\title{
La dea Eos e il trasporto di Memnone tra mito ed epica
}

La déesse Éos et le transport de Memnon entre mythe et poésie épique

Ilaria Sforza

\section{(2) OpenEdition}

12 Journals

Edizione digitale

URL: https://journals.openedition.org/gaia/281

DOI: 10.4000/gaia.281

ISSN: 2275-4776

\section{Editore}

UGA Éditions/Université Grenoble Alpes

\section{Edizione cartacea}

ISBN: 978-2-37747-057-0

ISSN: 1287-3349

Notizia bibliografica digitale

Ilaria Sforza, «La dea Eos e il trasporto di Memnone tra mito ed epica», Gaia [Online], 21 | 2018, online dal 01 novembre 2018, consultato il 09 décembre 2021. URL: http://journals.openedition.org/gaia/281 ; DOI: https://doi.org/10.4000/gaia.281

Questo documento è stato generato automaticamente il 9 décembre 2021.

Gaia. Revue interdisciplinaire sur la Grèce archaïque 


\title{
La dea Eos e il trasporto di Memnone tra mito ed epica
}

La déesse Éos et le transport de Memnon entre mythe et poésie épique

\author{
Ilaria Sforza
}

1 Nella cospicua serie di immagini che ritraggono Eos in movimento sui vasi greci del V secolo a.C., è possibile distinguere tre grandi temi: Eos come divinità che porta, al suo apparire nel cielo, la luce del giorno; Eos che insegue e talvolta "rapisce" un giovane; Eos che solleva in volo il corpo del figlio' ${ }^{1}$.

2 Tutte queste immagini - a differenza del motivo del duello tra Achille e Memnone inquadrati dalle rispettive madri, già attestato sull'arca di Cipselo ${ }^{2}-$ presentano, pur con le loro molteplici peculiarità, un tratto in comune: Eos è resa come una figura in movimento. Le ali, attributo frequente della dea nelle scene di inseguimento di un giovane e/o di trasporto del morto, sembrano sottolineare l'efficacia dell'azione divina.

3 Prima di approfondire il significato di immagini molto studiate ma che restano a tutt'oggi discusse come il celebre tondo della coppa di Duride (fig. 6$)^{3}$, in cui Eos è raffigurata come una dea alata che solleva il corpo nudo di Memnone, riteniamo necessario risalire alle origini di questa divinità, partendo dalla sua genealogia nelle fonti epiche.

\section{Eos e i venti}

Esiodo fa discendere la dea dell'apparire del giorno dai titani Theia e Iperione insieme a Elios e Selene, che sono quindi suoi fratelli ${ }^{4}$ :

Theia Elios grande e la splendida Selene

e Eós, che a tutti i mortali risplende

e agli immortali dèi che possiedono l'ampio cielo,

generò, giacendo con Iperione in amore. (Esiodo, Teogonia, 371-374)

Inoltre, è a questa dea in unione con Astreo, figlio del titano Creio e di Eurybie, nata da Ponto e Gaia, che Esiodo attribuisce la maternità degli anemoi "dall'animo forte", Zefiro, 
che porta il sereno, Borea dal rapido corso, e Noto ${ }^{5}$. Come è stato osservato, questa genealogia definisce diversi gradi di luminosità: a livello fisico la luce nitida del mattino (Eos) e la luce notturna degli astri - nei versi successivi, infatti, Esiodo afferma che dopo i venti la dea del mattino generò l'astro Lucifero e le stelle risplendenti ${ }^{6}-$, a livello atmosferico la luminosa provenienza dello spirare dei venti?

Nell'Inno omerico a Elio, Eos viene fatta discendere dal titano Iperione e da Eurifaessa "dagli occhi di giovenca"s, mentre in Eschilo Eos è figlia di Eufrone, identificabile con Notte ${ }^{9}$. A quest'ultima attribuisce la maternità di Eos Quinto Smirneo, autore di una continuazione dell'Iliade databile con ogni probabilità tra la seconda metà del II e la fine del III secolo d.C. ${ }^{10}$, in cui l'episodio della morte di Memnone, che era uno dei temi centrali dell'Etiopide di Arctino di Mileto, poema epico ciclico databile verso la fine dell'VIII secolo a.C. ${ }^{11}$, vede come protagonisti del trasporto dell'eroe morto i Venti, suoi fratelli secondo la già ricordata genealogia esiodea ${ }^{12}$ :

Veloci tutti insieme i Venti

per ordine della madre si muovevano per un'unica via

verso la piana di Priamo e si sparsero intorno al morto:

velocemente quelli presero il figlio di Eos

e lo portarono nell'aere lucente; era afflitto il loro

animo per il fratello caduto, e intorno l'etere

gemeva. (Quinto Smirneo, II, 550-556)

7 La funzione svolta qui dai Venti di trasportare il corpo di Memnone li pone, pertanto, in stretta relazione con i gemelli generati per partenogenesi dalla Notte secondo la Teogonia esiodea (211-213) ${ }^{13}$, Hypnos e Thanatos, i quali svolgono nell'Iliade la funzione analoga di trasportare in Licia il corpo del morto Sarpedone, affidato loro da Apollo ${ }^{14}$ :

E subito [Apollo] portò l'illustre Sarpedone fuori

tiro, lo portò lontano e lo lavò nella corrente del fiume,

lo unse d'ambrosia e gli mise addosso vesti immortali;

poi lo affidò ai portatori veloci,

il Sonno e la Morte, i gemelli, che subito

lo deposero nella ricca regione di Licia. (Omero, Iliade, XVI, 678-683)

8 Nell'Iliade l'aurora è il momento in cui appare la luce del giorno: l'arrivo della luce è rappresentato attraverso l'immagine della dea, che si leva dal letto di Titono o che sorge dalle correnti dell'Oceano ${ }^{15}$. Almeno in due scene, tuttavia, l'apparire dell'aurora segna un momento ben preciso, quello della raccolta delle ossa del morto dopo lo spegnimento della pira funebre ${ }^{16}$. La scena si ripete, con alcune significative variazioni, nella descrizione dei funerali di Patroclo nel canto ventitreesimo e di quelli di Ettore nel ventiquattresimo; nonostante gli elementi di analogia tra i due rituali descritti, sorprende la varietà delle espressioni adoperate nei due brani ${ }^{17}$. Nella prima scena, la più estesa, che riportiamo di seguito, l'arrivo dell'Aurora è preceduto dall'apparire di Eosphoro, Lucifero, ricordato solo qui in Omero e figlio della dea in Esiodo ${ }^{18}$. Inoltre, all'apparire di Eos, il rogo si spegne e i venti tornano a casa. Solo allora il sonno sopraggiunge a dare sollievo al Pelide afflitto per la morte del compagno ${ }^{19}$ :

Quando la stella del mattino annuncia la luce

sopra la terra, e sul mare si stende l'aurora dal peplo dorato,

allora si esaurì il rogo e si spense la fiamma.

I venti tornarono alla loro casa sul mare

di Tracia, ed esso gemeva gonfiandosi.

Dall'altra parte del rogo il figlio di Peleo

si coricò esausto e gli volò addosso il dolce sonno.

(Omero, Iliade, XXIII, 226-232) 
Segue la raccolta delle ossa di Patroclo che vengono deposte dai compagni in un'urna d'oro, dove, alla morte di Achille, esse verranno custodite insieme a quelle dell'amico ${ }^{20}$. Nel brano similare ma più sintetico dei funerali di Ettore, il momento in cui il rituale ha luogo viene semplicemente indicato per mezzo del verso formulare «quando al mattino riapparve l'Aurora dita di rosa ${ }^{21}$, senza alcun accenno a Lucifero, né al calare dei venti con l'apparire del giorno. Dovremo pertanto ritenere eccezionale, nel primo dei due passi, l'associazione a Eos di Eosphoro, suo figlio, e di Hypnos che può considerarsi, a partire da alcune fonti antiche che ritengono anche Eos figlia di Nyx, fratello dell'Aurora $^{22}$. Il legame tra il sorgere dell'Aurora e il ritirarsi dei venti da una parte, lo spegnimento della pira funebre e la raccolta delle ossa dell'eroe dall'altra, sembra suggerire, quindi, una possibile spiegazione del ruolo di portatori attribuito dalle fonti, letterarie e iconografiche, alternativamente a Hypnos e Thanatos o ai Venti stessi. Entrambe le coppie di figure gemellari, munite di ali nell'iconografia vascolare ${ }^{23}$, sono da mettere in relazione con Eos e con altre figure femminili alate come Iris. Infatti, nel passo omerico relativo ai funerali di Patroclo, Achille invoca Zefiro e Borea poiché la pira funebre di Patroclo stenta a prendere fuoco ${ }^{24}$. L'intervento dei Venti, tuttavia,

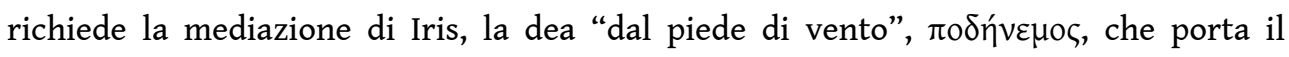
messaggio ai Venti, occupati a banchettare in casa dell'impetuoso Zefiro ${ }^{25}$.

10 Dunque, le ali, che caratterizzano i venti nelle raffigurazioni vascolari, sembrano rimarcare lo status divino di queste entità, come nelle scene del rapimento di Oreithyia da parte di Borea $^{26}$, qualificandole come manifestazioni della potenza divina, talvolta antropomorfizzate in figure femminili mostruose come le Arpie, di cui viene enfatizzata nelle fonti letterarie la velocità e, come vedremo tra breve, la funzione rapitrice ${ }^{27}$. D'altronde, l'affinità tra la divina Iris e i venti è suggerita in Esiodo dalla

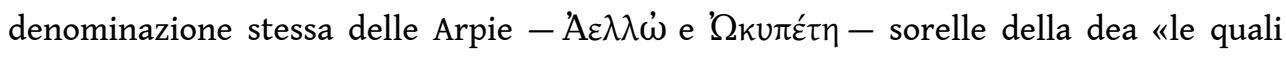
sanno seguire il soffio dei venti e gli uccelli nel volo / con le ali veloci: tanto rapide infatti si slanciano» ${ }^{28}$. A un esame dei passi esiodei, inoltre, emerge come le Arpie, che il poeta di Ascra per primo ci descrive alate, fossero già stabilmente associate alla sfera semantica della velocità, cui rimandano i diversi appellativi di queste entità divine nelle fonti antiche ${ }^{29}$. In Omero, la loro funzione è costantemente espressa dall'aoristo $\dot{\alpha} v \eta \rho \varepsilon i ́ \psi \alpha v \tau 0^{30}$, al punto che è stata ipotizzato, sulla base dell'allitterazione, un collegamento etimologico tra il loro nome collettivo Apruı 1 e questa forma del verbo, impiegata nelle fonti per esprimere l'azione di divinità o di fenomeni naturali come le tempeste, anche se la connessione generalmente suggerita dalla critica è con $\dot{\varepsilon} \rho \varepsilon ́ \pi \tau o \mu \alpha 1$, "strappare", "divorare", detto di animali che mangiano erba o vegetali ${ }^{31}$.

11 Proprio il tema del rapimento, associato anche a Eos nel mito, offre ulteriori prospettive di indagine sul significato della serie di raffigurazioni vascolari in cui la dea alata trasporta il corpo di Memnone. Il legame genealogico e simbolico tra Eos e i Venti, che abbiamo fin qui delineato, trova riscontro anche in alcune scene di inseguimento che vedono protagonisti rispettivamente Eos e Borea su vasi provenienti da un medesimo contesto funerario. Il motivo del rapimento di Oreithyia, attestato sul lato A di un celebre stamnos a figure rosse ${ }^{32}$ proveniente dalla Tomba di Brygos II a Capua e databile verso il 470-460 a.C. che presenta sul lato B l'inseguimento di un giovane da parte di una figura femminile alata interpretabile come Eos che insegue Cefalo, risulta già attestato sull'arca di Cipselo ${ }^{33}$. Qui Borea presenta, oltre alle ali, delle "code di serpente" al posto dei piedi. Se si presta fede all'identificazione del personaggio alato con Borea proposta dal periegeta - confortata da scene vascolari in cui l'entità divina è 
similmente dotata di code di serpente -, non sfuggirà il valore iniziatico dell'episodio raffigurato, che presenta forti analogie con il rapimento di Core da parte di Ade, dio degli Inferi. Le scene che mostrano il rapimento di una fanciulla da parte di un dio alludono infatti, generalmente, al cambiamento di status della fanciulla medesima, un tema iniziatico, quello del passaggio dalla pubertà all'età adulta, contenuto più o meno esplicitamente in tutte le fonti letterarie a noi note del mito di Core ${ }^{34}$.

Alla luce dell'associazione tra il rapimento di Core e l'inseguimento di Cefalo sullo stamnos proveniente da Capua, passiamo ora a riflettere sul significato del "rapimento" di un mortale da parte della dea del mattino, motivo che ebbe particolare fortuna nella ceramica vascolare del V secolo a.C. e che risulta ben attestato nelle fonti letterarie a partire dall'epica omerica ${ }^{35}$.

\section{Il rapimento di Titono, Orione, Clito e Cefalo}

Nell'Odissea, accanto all'impiego ricorrente del verso formulare che indica con l'arrivo dell'Aurora l'apparire della luce del giorno ${ }^{36}$, troviamo per la prima volta un riferimento a Memnone, il "figlio splendido dell'Aurora lucente" che uccise Antiloco ${ }^{37}$. Esiodo lo menziona in un passo della Teogonia in cui ricorda le diverse unioni di Eos con giovani mortali e la loro progenie $e^{38}$ :

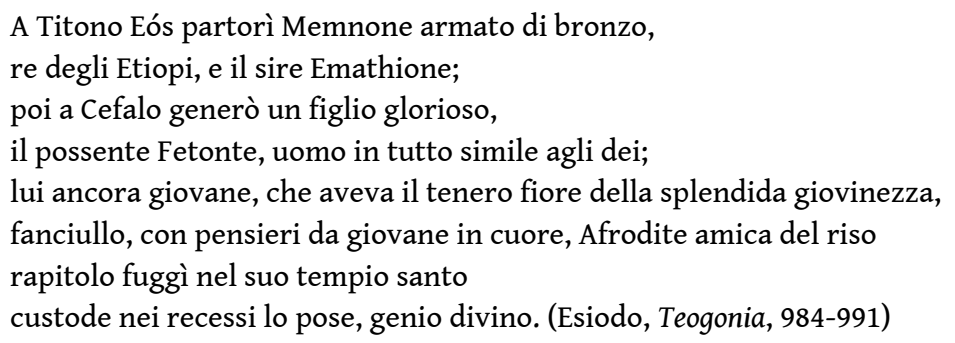

Dall'unione con Titono, dunque, Eos ebbe due figli, Memnone, re degli Etiopi, il cui nome è riconducibile a $\mu$ í $\mu v \omega$ e significa pertanto "colui che sta saldo", come suggerisce

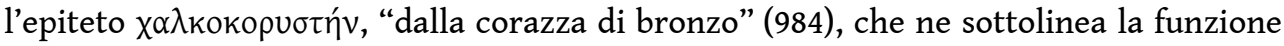

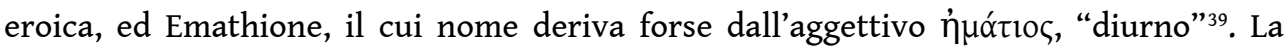
seconda unione ricordata qui da Esiodo è quella con Cefalo, da cui nacque Fetonte, rapito dalla dea Afrodite proprio nel fiore degli anni, quando era ancora un $\pi \alpha \tilde{\imath} \varsigma$, per

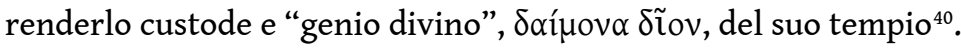

Nelle fonti letterarie, tuttavia, è Eos la divinità femminile cui è attribuito più di frequente l'atto di "rapire" un giovane mortale a causa della sua bellezza ${ }^{41}$. Un'allusione all'amore di Eos e Titono è contenuta nell'emistichio formulare, già attestato nell'Iliade, «L'Aurora si alzò dal letto dell'illustre Titone / per portare la luce ai mortali e agli immortali $»^{42}$. Lo stesso episodio è narrato in un ampio passaggio nell'Inno omerico ad

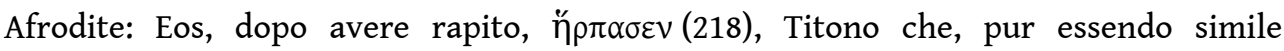

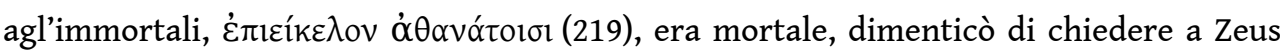
per il suo sposo, oltre all'immortalità, "che gli tenesse lontana l'odiosa vecchiaia" ${ }^{43}$. Di conseguenza ${ }^{44}$,

[...] fin quando egli era nella molto amabile giovinezza, godendo l'amore dell'Aurora dai fiori d'oro, che sorge di buon mattino, dimorava presso le correnti dell'Oceano, ai confini della terra: ma quando le prime ciocche bianche scesero giù dal bel capo e dal nobile mento, 
dal suo letto si astenne l'Aurora veneranda;

tuttavia, tenendolo nelle sue stanze, lo nutriva

di cibo terreno e di ambrosia, e gli donava belle vesti.

Ma quando con tutto il suo peso gravò su di lui l'odiosa vecchiaia

ed egli non riusciva più a muovere né a sollevare le membra,

questa nel suo animo le sembrò la decisione migliore:

lo relegò all'interno della casa, e serrò su di lui le porte risplendenti.

(Inno omerico, V, 225-236)

\section{l'immortalità per l'amato Titono, una richiesta esaudita solo in parte, dal momento che} la dea non aveva chiesto per il suo amato anche l'eterna giovinezza. Una seconda volta, troviamo Eos nell'atto di intercedere presso Zeus per ottenere l'immortalità di un uomo, suo figlio Memnone, come riferisce Proclo nel riassunto dell'Etiopide: «Poi Achille uccide Memnone: e per lui Eos, per intercessione di Zeus, ottiene l'immortalità» ${ }^{45}$. In secondo luogo, è bene considerare con quali espedienti la dea cerchi di preservare il corpo di Titono dal disfacimento inesorabile causato dalla vecchiaia: lo nutre di cibo

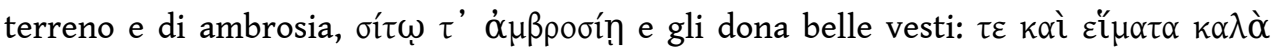
$\delta ı \delta o v ̃ \sigma \alpha$. Le azioni compiute dalla dea sono simili a quelle che altre divinità - Apollo, Teti e Afrodite - compiono nell'Iliade allo scopo di preservare la bellezza del corpo di un eroe dopo la morte: Apollo unge di ambrosia il corpo di Sarpedone per poi consegnarlo ai portatori divini, Sonno e Morte, restituendo così all'eroe, reso irriconoscibile dalla polvere e dalle ferite ricevute, un aspetto degno degli onori che gli verranno tributati in Licia da compagni e parenti ${ }^{46}$. Analogo è il trattamento riservato da Teti, su richiesta di Achille, al corpo di Patroclo che comincia a dare segni di decomposizione: la dea istilla nelle narici del guerriero morto nettare e ambrosia, perché il corpo resti intatto ${ }^{47}$. Infine, il cadavere di Ettore è unto da Afrodite con olio di rose ambrosio, mentre Apollo impedisce, con una nube plumbea, che l'ardore del sole ne dissecchi la pelle ${ }^{48}$ :

[...] [i cani] non si curavano di Ettore: li allontanava la figlia di Zeus Afrodite

e lo ungeva giorno e notte con olio di rose,

immortale, perché Achille non lo scorticasse tirandolo.

Apollo portò dal cielo sulla pianura una nube

oscura, e coprì tutto quanto il terreno

occupato dal morto, perché l'ardore del sole

non seccasse la pelle attorno ai muscoli e ai tendini.

(Omero, Iliade, XXIII, 185-191)

A compiere queste operazioni, volte a preservare il corpo dell'eroe dalla contaminazione provocata dalla polvere e dal sangue delle ferite, sono dunque nell'Iliade divinità femminili come Afrodite e Teti, cui si aggiunge Apollo ${ }^{49}$. L'importanza delle figure divine femminili in un contesto eroico risalta nelle rappresentazioni del duello di Achille e Memnone inquadrati dalle loro madri. Il motivo delle due madri che assistono al duello, talvolta associato nelle immagini alla psicostasia, nucleo centrale dell'omonima tragedia di Eschilo per noi perduta, doveva corrispondere nell'Etiopide, per quanto è possibile desumere dal resoconto di Proclo, alla richiesta di rendere immortali i propri figli rivolta a Zeus dalle due de $e^{50}$. Entrambe ottennero che i corpi degli eroi, dopo le cerimonie funebri, venissero trasportati lontano; nel caso di Achille, riferisce Proclo, «Teti, rapendo il figlio dalla pira, lo trasporta nell'isola di Leucade» ${ }^{51}$. 


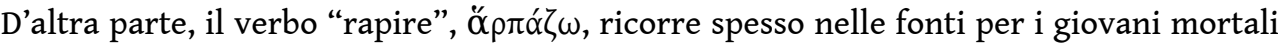
di cui Eos si innamora: Clito nell'Odissea ${ }^{52}$; Titono nell'Inno omerico ad Afrodite ${ }^{53}$; Cefalo nell'Ippolito di Euripide e nella Biblioteca dello pseudo-Apollodoro ${ }^{54}$. Secondo una notizia riportata da quest'ultimo, Eos era stata punita da Afrodite con un appetito sessuale insaziabile per avere sedotto Ares ${ }^{55}$. La dea è ricordata nell'Odissea anche per il rapimento di Orione, celebre cacciatore di cui si era invaghita a causa della sua bellezza ${ }^{56}$. Il racconto dell'amore di Eos per il giovane e bellissimo Orione è nella riposta di Calipso all'ordine di Zeus di lasciar andare Odisseo, riferitole da Ermes: il paragone istituito così da Calipso tra sé stessa ed Eos fa riflettere sul significato dell'isola di Ogigia, ai confini del mondo, dove l'offerta dell'immortalità a Odisseo, che l'eroe prontamente rifiuta, richiama altri paradigmi mitici, come il dono dell'immortalità concesso da Zeus a Titono prima e a Memnone poi per intercessione di $\operatorname{Eos}^{57}$.

Oltre a Orione, anche Clito, figlio di Mantio, venne rapito da Eos «per la sua bellezza» ${ }^{58}$. Nel passo omerico è inoltre esplicitato lo scopo dell'azione compiuta dalla dea: «affinché vivesse tra gli immortali». L'emistichio formulare ricorre nell'Inno omerico ad Afrodite per Ganimede rapito da Zeus: «In verità, il saggio Zeus rapì il biondo Ganimede / per la sua bellezza, affinché vivesse tra gli immortali» ${ }^{59}$.

Nell'Inno omerico, l'inspiegabile scomparsa del giovinetto provoca la disperazione del padre, «che non sapeva dove il turbine divino gli avesse rapito suo figlio» ${ }^{60}$. Troo, infatti, non sapendo che Zeus aveva destinato Ganimede al nobile compito di «versare da bere agli dei - prodigio a vedersi, onorato da tutti gl'immortali-», attribuisce la misteriosa scomparsa del figlio a un "turbine divino", $\theta \varepsilon ́ \sigma \pi ı \varsigma$ ö $\varepsilon \lambda \lambda \alpha$, finché Zeus stesso, mosso a pietà dal dolore del padre, come compensazione per la perdita del figlio, gli

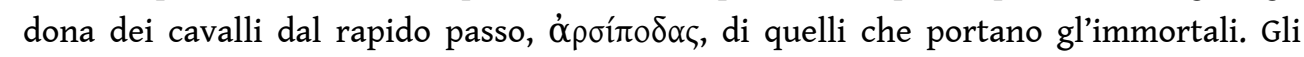
spiega, inoltre, che Ganimede era «immortale e immune da vecchiezza, come gli dei»,

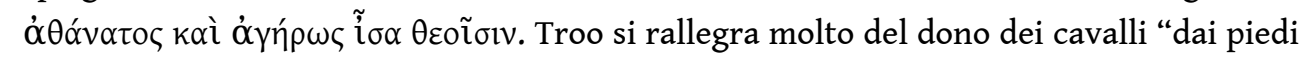
veloci", $\dot{\alpha} \varepsilon \lambda \lambda \circ \pi_{0} \delta \varepsilon \sigma \sigma l v$ - l'aggettivo è un composto di ŏ $\varepsilon \lambda \lambda \alpha$, "turbine" - come risarcimento per la perdita del figlio.

21 Il rapimento di Ganimede, che presenta diverse analogie con i rapimenti compiuti da Eos, offre alcuni spunti di riflessione: in primo luogo, la scomparsa di Ganimede è causa di dolore immenso per suo padre come, nell'Inno a Demetra, la scomparsa di Persefone per la madre Demetra, che inizia così la disperata ricerca della figlia ${ }^{61}$. Anche Persefone, del resto, era stata rapita contro la sua volontà ${ }^{62}$. In secondo luogo, la causa della scomparsa di Ganimede, avvertita come evento luttuoso, è attribuita da Troo a un turbine di vento, ő́ $\varepsilon \lambda \lambda \alpha$, conformemente alla consuetudine omerica.

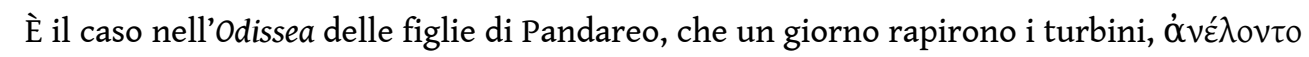
$\theta$ Úc $\lambda \lambda \alpha{ }^{63}{ }^{63} ;$ poco dopo viene detto che a rapire le fanciulle furono le Arpie che le diedero in balia delle Erinni odiose ${ }^{64}$. L'episodio, peraltro poco conosciuto, delle figlie di Pandareo, viene citato da Penelope nel passo omerico per augurarsi che i numi la annientino, come fecero con queste fanciulle e le consentano, facendola scendere sotto la terra odiosa, di rivedere Odisseo, suo sposo ${ }^{65}$. L'impiego in questi versi del verbo "annientare", óı $\sigma \tau o ́ \omega$, nel senso di "diventare invisibile", come conseguenza del "venire rapiti dai turbini o dalle Arpie", non appare casuale, alla luce del confronto con altri passi odissiaci, in cui l'eventualità del rapimento da parte di un turbine è sinonimo dello scomparire in mare travolto da una tempesta ${ }^{66}$. Questo tipo di morte appare, a differenza dell'uccisione in battaglia, del tutto disonorevole, poiché priva la vittima del 
klèos, unico mezzo per gli eroi omerici di raggiungere l'immortalità ${ }^{67}$. Il concetto è ben espresso da Telemaco che si lamenta della scomparsa del padre spiegando ${ }^{68}$ :

Ché della sua morte non avrei tanto strazio, se tra i compagni fosse caduto, in terra dei Teucri, o tra le braccia dei suoi, dopo aver dipanato la guerra: tomba gli avrebbero fatto tutti insieme gli Achei, e al figlio gran fama avrebbe acquistata in futuro. Invece l'hanno travolto ( $\dot{\alpha} v \eta p \varepsilon i ́ \psi \alpha v \tau o)$ le Arpie, senza gloria ( $\dot{\alpha} \kappa \lambda \varepsilon \mid \tilde{\omega} \varsigma)$;

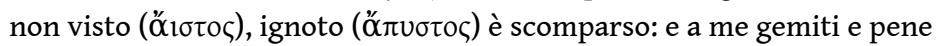
ha lasciato. (Omero, Odissea, I, 236-243)

Viene così a delinearsi un'alternativa tra il "rapimento" di un mortale - spesso compiuto da Eos, controparte femminile di Zeus/Ade - e la belle mort seguita dagli onori funebri commisurati alle imprese eroiche compiute in vita riservata ai guerrieri omerici che, come Achille, rinunciando a una vita lunga e anonima, ottengono in premio il klèos àphthiton ${ }^{69}$.

\section{Il trasporto dell'eroe dopo la morte: Sarpedone e Memnone}

È opportuno, a questo punto, riflettere sul rapporto che intercorre, nell'epica omerica, tra due temi che paiono talvolta intersecarsi, la scomparsa di un giovane nel fiore degli anni e la morte in guerra di un eroe dal valore straordinario. I due temi sono contrapposti nelle parole rivolte da Zeus a Era nell'imminenza del duello decisivo tra Sarpedone e Patroclo ${ }^{70}$ :

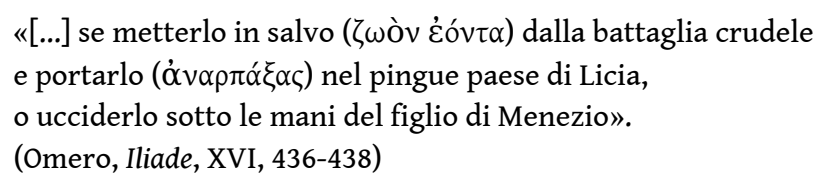

La prima alternativa paventata da Zeus, quella cioè di strappare alla morte il figlio, «un uomo mortale, da tempo dovuto al destino $)^{71}$, non è considerata accettabile da Era, che invita il suo sposo a riflettere sulle conseguenze che una simile scelta avrebbe: altri dei vorranno salvare i propri figli e proveranno sdegno per il privilegio concesso da Zeus a Sarpedone. La soluzione prospettata da Era, dunque, rappresenta una via di mezzo tra morte e immortalità: dopo aver lasciato morire Sarpedone sotto le mani di Patroclo, non appena il respiro e la vita lo abbiano lasciato, lo prendano la Morte e il Sonno soave e lo portino nell'ampia Licia, dove fratelli e compagni possano onorarlo come merita con una tomba e una stele: questo - conclude la dea - è l'onore dei morti ${ }^{2}$ !

L'unicità della scena ha avvalorato per molto tempo l'ipotesi, sostenuta da alcuni studiosi, di una derivazione del personaggio di Sarpedone nell'Iliade dalla figura di Memnone nell'Etiopide $e^{73}$. Tralasciando queste teorie di scuola neo-analitica a sostegno della presunta anteriorità dell'Etiopide rispetto all'Iliade, ormai in buona parte superate, quello che a noi preme considerare è l'ideologia eroica che emerge dalla risposta di Era, con cui sembra risolversi l'apparente dicotomia tra immortalità condizionale concessa ai giovani mortali rapiti dagli dei e morte in guerra di eroi semidivini trasportati altrove dopo la morte ${ }^{74}$.

Sul versante figurativo, le scene di trasporto del morto Sarpedone sui vasi attici da parte delle divinità gemellari Hypnos e Thanatos sono state oggetto di particolare 
attenzione negli ultimi decenni ${ }^{75}$. Il tema del trasporto del morto, generalmente nudo, da parte di due portatori, talvolta dall'aspetto giovanile, privi di barba e dotati di ali, talvolta dall'aspetto più maturo e spesso differenziati, come sull'arca di Cipselo - dove il colore distingueva il benigno Hypnos dalla funesta Thanatos $-{ }^{76}$, ha trovato una straordinaria popolarità sui vasi greci del V secolo a.C., poiché si prestava a rappresentare il corpo eroico in tutto il suo splendore, nel momento della belle mort ${ }^{77}$. All'interno della più ampia serie di rappresentazioni del trasporto del morto nella ceramica vascolare, e in rapporto alle immagini di Sarpedone, sono state commentate dagli studiosi anche le raffigurazioni di Eos alata che porta Memnone ${ }^{78}$.

Fin dalle sue più antiche attestazioni, nella ceramica attica a figure nere, il motivo del trasporto di Memnone presenta elementi di contiguità con altri due motivi, quello del rapimento e quello del compianto funebre.

Nella più antica scena di trasporto di Memnone da parte di Eos con i nomi dei personaggi iscritti, su un'anfora a figure nere databile tra il 510 e il 500 a.C. ${ }^{79}$, Eos alata, verso destra, rivolta indietro, porta il corpo nudo di Memnone in braccio come si trattasse di un bambino ${ }^{80}$. Uno schema analogo si ritrova nelle raffigurazioni del rapimento di Titono o di Cefalo da parte di Eos su alcuni vasi a figure rosse databili verso la metà del V secolo a.C. (fig. 1) ${ }^{81}$. Come si è avuto modo di osservare, nelle fonti epiche, l'azione del "rapire", arpàzein, un mortale da parte di una divinità, è interpretabile come un tentativo di consentire al giovane, dalla bellezza eccezionale, un superamento della propria condizione di mortalità. Questo tipo di "scomparsa", tuttavia, è valutata negativamente nei poemi omerici, dove al rapimento si contrappone il trasporto dell'eroe, dopo la morte, in un luogo marginale, perché al suo corpo possa essere data degna sepoltura e al suo nome una gloria immortale.

Fig. 1. - Lekythos a figure rosse, verso il 470 a.C.

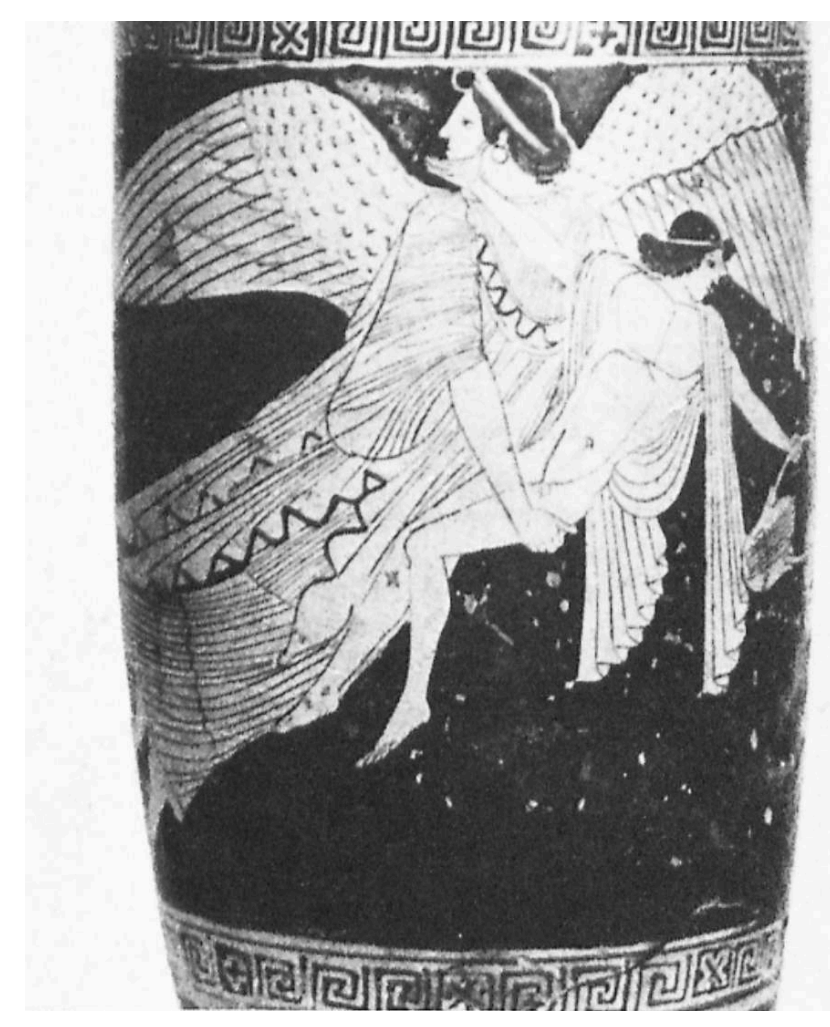

Madrid, Museo Arqueológico Nacional 11158. Da: LIMC, III (2), 1986, Eos 268, p. 577. 
Accanto alla valenza funeraria delle scene di "rapimento" - suggerita dal ritrovamento di alcuni vasi, come lo stamnos da Capua discusso sopra, in contesti sepolcrali - si riscontra in molte di queste scene l'aspetto giovanile, quasi infantile, delle figure maschili "rapite", un tratto che accomuna alcune raffigurazioni di "trasporto" del morto da parte di Hypnos e Thanatos e di Eos alata ${ }^{82}$. Su un olpe a figure nere databile verso il 500-490 a.C., per esempio, Eos alata chinata a destra solleva un giovane nudo, le cui braccia pendono verso il basso e il cui viso è rivolto verso il terreno (fig. 2$)^{83}$.

Fig. 2. - Olpe a figure nere, verso il 500-490 a.C.

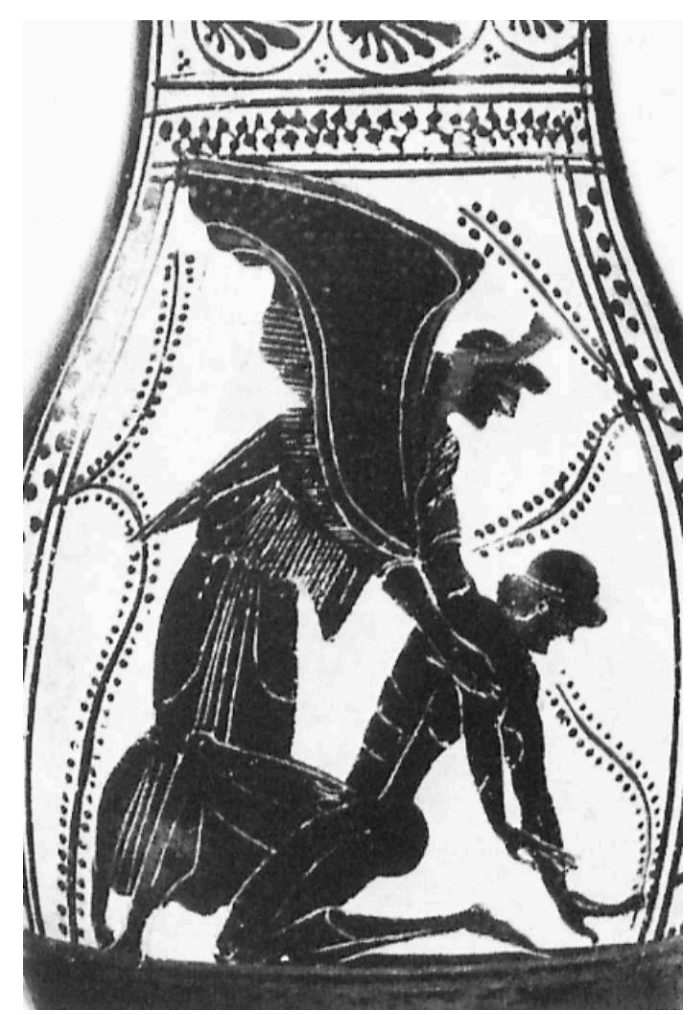

Bochum, Ruhr-Universität, Kunstsammlungen S494. Da: LIMC, III (2), 1986, Eos 318, p. 581

Analogamente, su uno skyphos a figure rosse dello stesso periodo, Eos è raffigurata mentre solleva un giovane nudo dall'aspetto giovanile, con gli occhi chiusi e le braccia che pendono inerti verso il terreno. Nella scena sono presenti altre due figure ai lati della coppia centrale: a sinistra Ermes, riconoscibile dal petaso e dal kerykeion, a destra una figura femminile non meglio identificata (fig. 3) ${ }^{84}$. Imberbe e con il volto reclinato all'indietro è anche il giovane morto trasportato da Eos su una pelike a figure rosse databile verso il 480 a.C. (fig. 4) 85 $^{85}$ le braccia del morto che pendono all'indietro, la sua bocca semiaperta e i dettagli anatomici del busto, solcato da una profonda ferita che sanguina, contribuiscono a rendere la scena più drammatica. In particolare, il contrasto tra il ricco panneggio di Eos e la nudità del corpo trasportato ricordano la rappresentazione più famosa di Eos e Memnone, con i nomi iscritti, nel tondo della coppa di Duride: in questa scena Eos alata, chinata verso destra, solleva il corpo del figlio nudo, con la barba e i capelli lunghi, il viso rivolto verso il terreno come in altre immagini (fig. 5) ${ }^{86}$. Dalla raffigurazione di Memnone come un eroe maturo, con il corpo solcato da profonde ferite che sanguinano copiosamente, si evince il carattere marcatamente epico del tema del trasporto dell'eroe in questa scena. Un'analoga 
insistenza sulle ferite del guerriero morto si riscontra anche nella scena del trasporto di Sarpedone da parte di Hypnos e Thanatos sul celebre cratere di Euphronios, di poco antecedente alla coppa di Duride (fig. 6) ${ }^{87}$. Le due figure gemellari alate, in questa immagine, portano sul capo un elmo corinzio sollevato, che ne indica la funzione di guerrieri; al medesimo contesto epico allude la presenza di due opliti stanti che inquadrano il gruppo centrale: a completare la scena Ermes al centro, in secondo piano, assiste al trasporto dell'eroe morto ${ }^{88}$.

Fig. 3. - Skyphos a figure rosse, verso il 525-475 a.C.

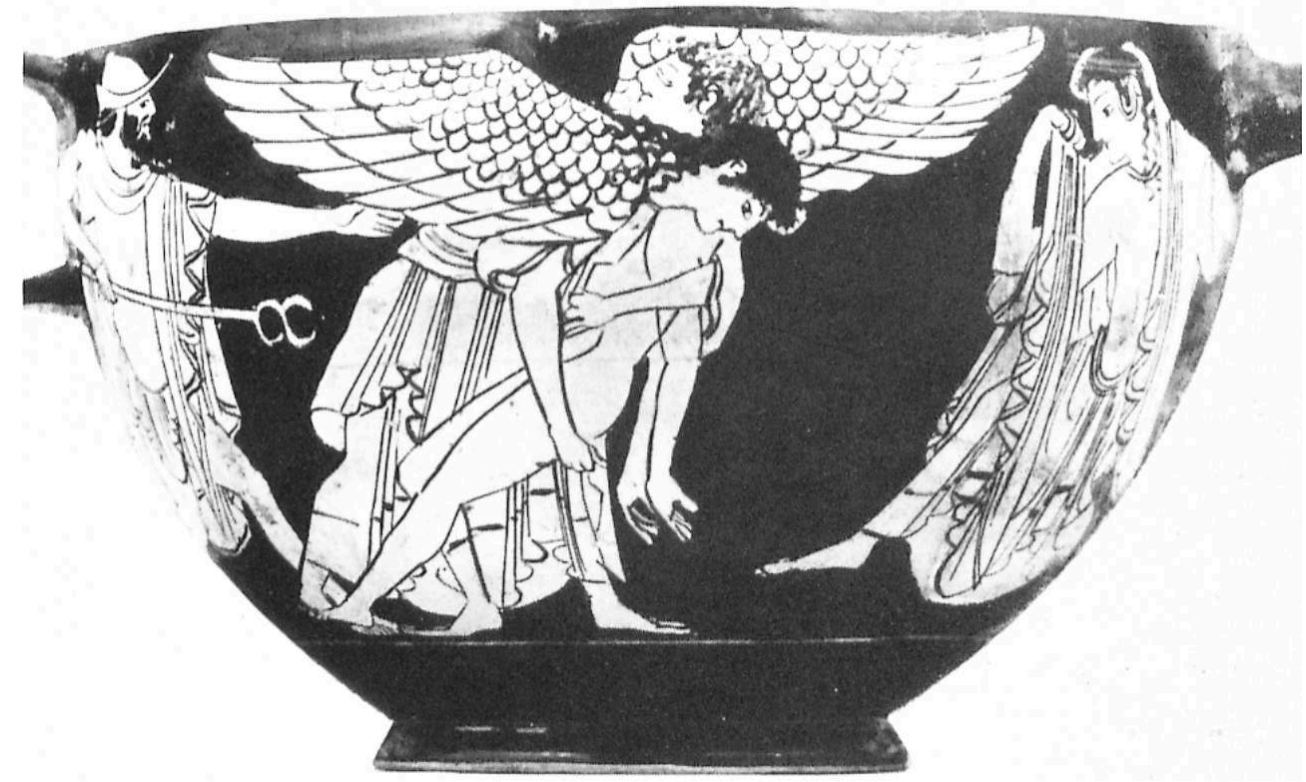

Berlin, Antikensammlung F2318. Da: LIMC, III (2), 1986, Eos 322, p. 581. 
Fig. 4. - Pelike a figure rosse, verso il 480 a.C.

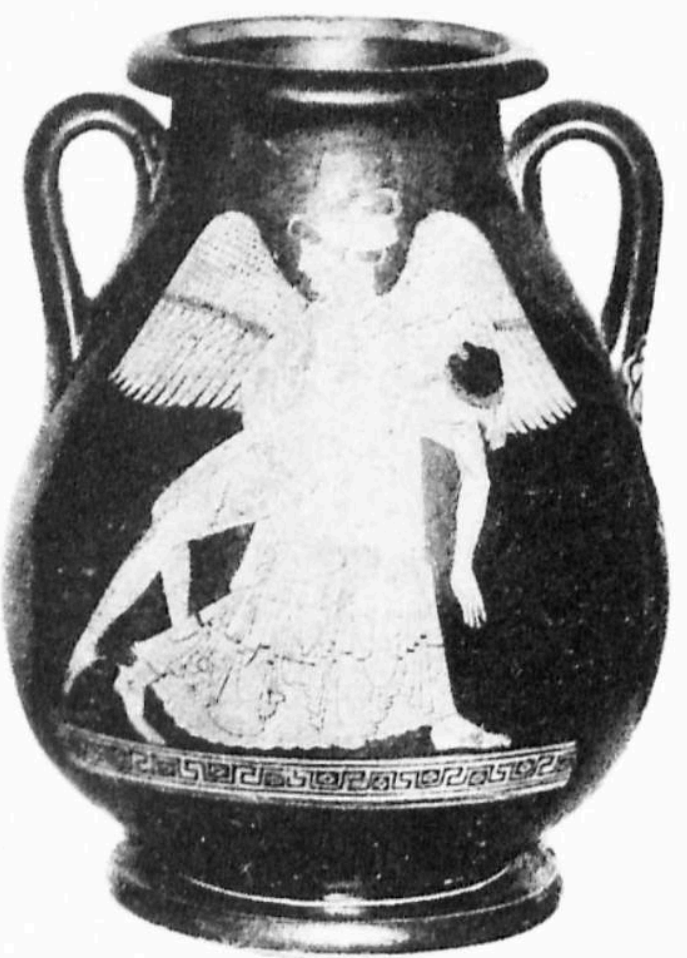

Paris, Musée du Louvre G232. Da: LIMC, III (2), 1986, Eos 332, p. 785).

Fig. 5. - Coppa a figure rosse del pittore Duride, verso il 485-480 a.C.

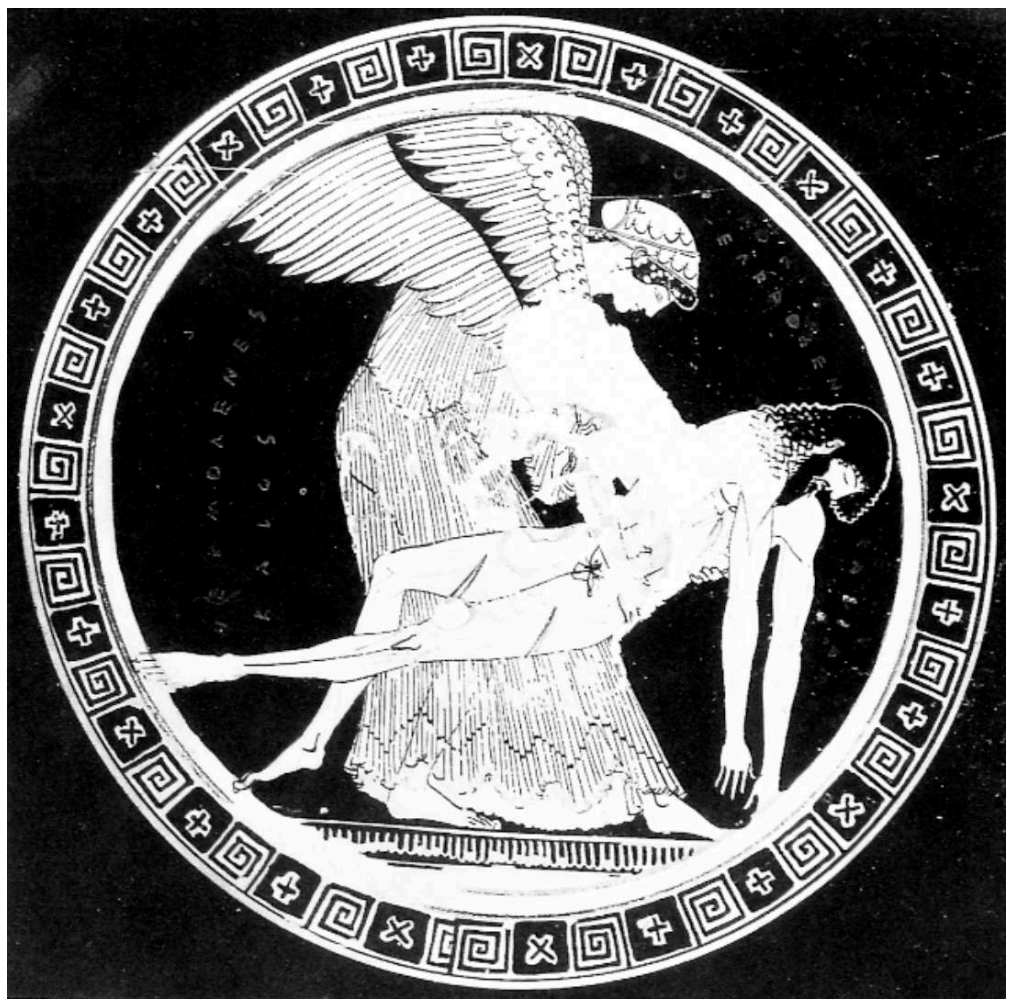

Paris, Musée du Louvre G115. Da: D. Buitron-Oliver (1995, n. 119, tav. 71). 
Fig. 6. - Cratere a figure rosse del pittore Euphronios, verso il 515-510 a.C.
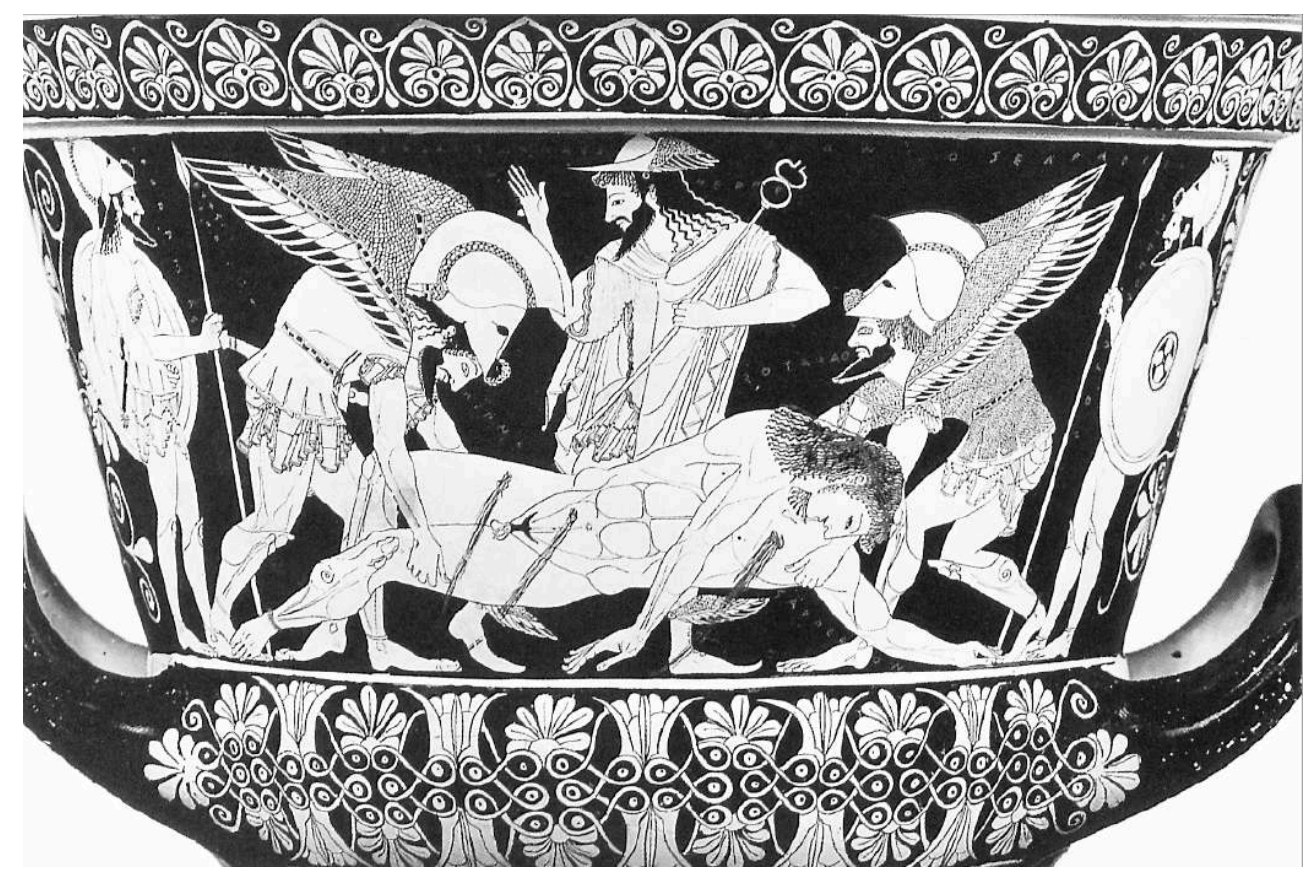

New York, MM 1972.11.10. Da: LIMC, VII (2), 1994, Sarpedon 4, p. 520.

Per tornare, dunque, alla funzione di Eos che trasporta il corpo di Memnone lontano dal campo di battaglia, occorre evidenziare il legame delle figure femminili, sui vasi attici, con il tema del compianto. In tal senso andrà intesa una scena unica, dipinta su un'anfora a figure nere del pittore del Vaticano (fig. 7) ${ }^{89}$, interpretabile, pure in assenza di iscrizioni, come il compianto di Eos sul corpo del figlio ${ }^{90}$. In piedi al centro dell'immagine una donna è raffigurata nell'atto di compiangere il morto strappandosi i capelli; allungato ai suoi piedi, su un letto di rami, il corpo di un eroe nudo con gli occhi chiusi; dietro la donna, a sinistra, una panoplia è disposta in verticale, appoggiata a due alberi ${ }^{91}$.

La presenza straordinaria di elementi di paesaggio in questa scena vascolare, nonché la raffigurazione di un uccello lungo l'asse verticale, proprio sopra la testa della donna, sembrano evocare una tradizione relativa al compianto di Memnone, riportata da Eliano e risalente forse già a Esiodo, secondo cui alcuni uccelli simili a falchi, chiamati "Memnoni" dal nome dell'eroe, si recherebbero ogni anno presso la tomba di Memnone per celebrarne la morte con una sorta di combattimento rituale ${ }^{92}$. Un'eco del compianto dell'eroe è nei versi di Quinto Smirneo riferiti al trasporto del suo corpo: dal sangue che cola copiosamente dal cadavere sollevato dai Venti si forma il fiume Paflagonio, le cui acque putride esalano vapori nauseabondi una volta l'anno ${ }^{93}$. 
Fig. 7. - Anfora a figure nere del pittore del Vaticano, verso il 540-520 a.C.

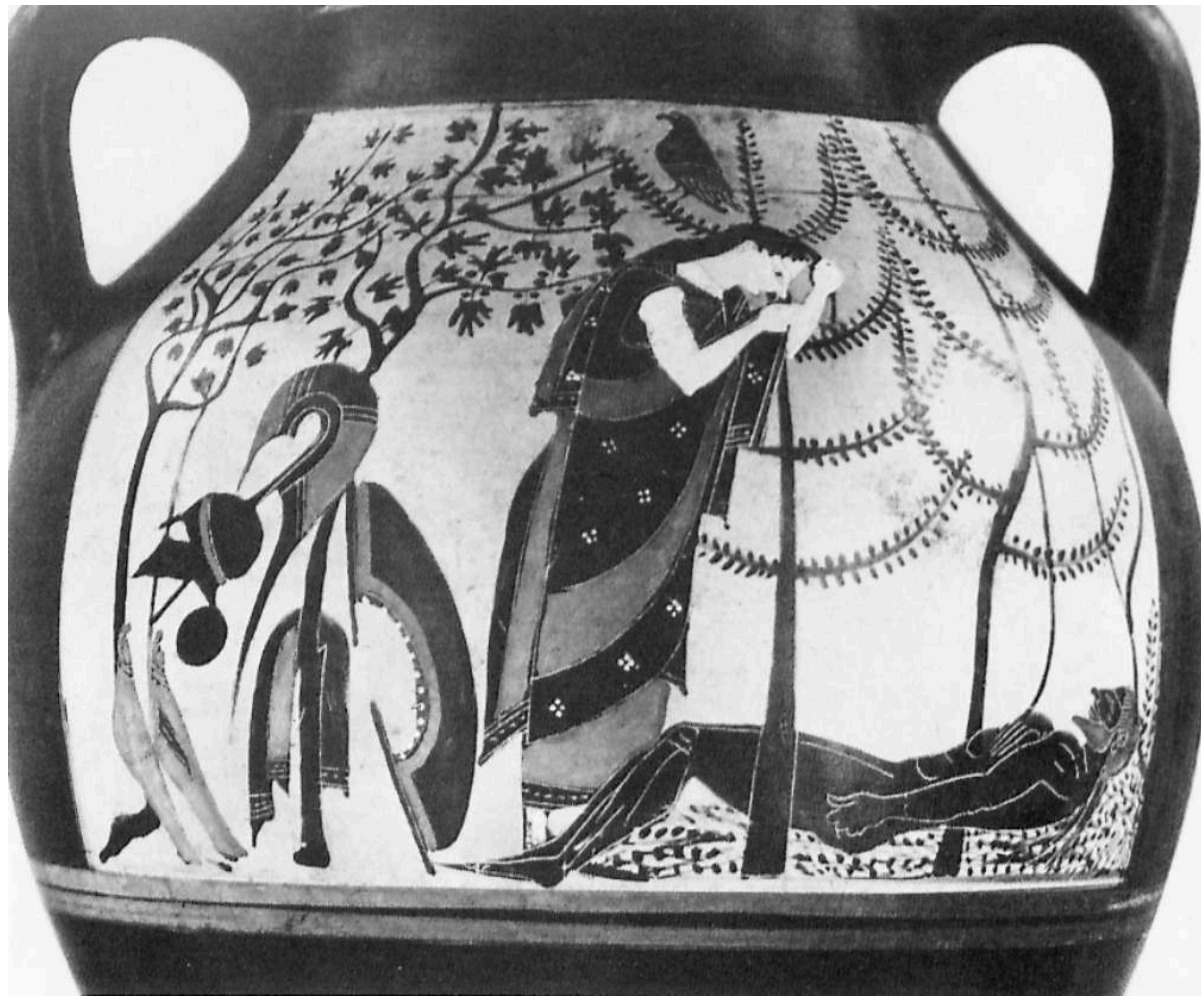

Vaticano 16589. Da: LIMC, III (2), 1986, Eos 327, p. 582. depongono ${ }^{94}$ :

[...]

presso le correnti del fiume Esepo dalle acque profonde, dove c'è il bosco delle Ninfe dalle belle chiome,

bello: poi eressero un grande tumulo

le figlie di Esepo, assai fitto di alberi

d'ogni specie; e molto le dee attorniarono di lamenti

il figlio di Erigenia dal bel trono, onorandolo. (Quinto Smirneo, II, 585-592)

Alla scomparsa dell'eroe, inoltre, fa seguito la scomparsa, altrettanto misteriosa, dell'esercito degli Etiopi i quali, dopo avere dato sepoltura al re, si trasformano in quegli uccelli che da lui prendono il nome di "Memnoni" e si battono sulla tomba dell'eroe sino a uccidersi tutti ${ }^{95}$ :

Prontamente i neri Etiopi Memnone,

piangendo, seppellirono; allora Erigenia dai grandi occhi,

loro che molto si dolevano attorno al tumulo del forte figlio,

li trasformò in uccelli e gli concesse di portarsi nell'aria.

Innumerevoli stirpi di mortali ora li chiamano

Memnoni, ché ancora sulla tomba del loro re

aggirandosi piangono, spargendo polvere sopra

il tumulo, suscitano poi confusione l'uno contro l'altro

per onorare Memnone... (Quinto Smirneo, II, 643-650)

36 Resta, in Quinto Smirneo, l'incertezza su dove sia stato portato il corpo di Memnone, se nelle case di Ade o tra i beati presso la pianura Elisia ${ }^{96}$ :

[...] ed egli nelle case di Ade

o tra i beati, sulla terra della piana Elisia, 
gioisce e si rasserena nell'animo l'immortale Eos

a vederli; essi si danno da fare finché sfiniti

si uccidono l'un l'altro nella mischia oppure

entrambe le parti compiono il loro destino gareggiando per il loro signore. (Quinto

Smirneo, II, 650-655)

La medesima collocazione del regno di Memnone ai confini dell'Oceano, dove si trovano pure, secondo Esiodo, la pianura Elisia e le Isole dei Beati, suggeriscono il profondo legame di questo eroe epico con il tema dell'immortalità ${ }^{97}$. D'altra parte, tale condizione è raggiunta solo a patto di affrontare, come tutti gli eroi epici, la morte in battaglia. Così, la trasformazione degli Etiopi in "Memnoni" appare legata, nell'immaginario antico, al compianto dell'eroe morto, come in questa descrizione di Pausania del dipinto che il celebre Polignoto di Taso, fiorito nella prima metà del V secolo a.C., realizzò per decorare la lesche degli Cnidi a Delfi ${ }^{98}$ :

Sulla clamide di Memnone sono ricamati degli uccelli: Memnonidi è il nome di questi uccelli e ogni anno, come affermano gli abitanti dell'Ellesponto, in giorni determinati essi vanno alla tomba di Memnone, spazzano tutta la parte del monumento funebre che è nuda d'alberi o d'erba e l'aspergono con le ali inumidite d'acqua dell'Esepo.

\section{Conclusioni}

Dall'esame delle fonti epiche e delle raffigurazioni vascolari relative alla dea Eos nella Grecia antica, è emersa una complessità di funzioni e di significati, che è possibile fare risalire alla fase culturale testimoniata dall'epica omerica ed esiodea. Nonostante la frammentarietà delle testimonianze del ciclo epico, è stato possibile ricostruire un profondo legame tra questa divinità e il tema del rapimento: infatti, Eos appare come la dea che "rapisce" giovani mortali - Titono, Orione, Clito, Cefalo - in una ricca serie di testimonianze, letterarie e iconografiche. Tutti questi giovani sono accomunati dalla loro eccezionale bellezza e dalla loro estrema giovinezza. È parsa pertanto probabile la connessione con il tema iniziatico, suggerita sia dai contesti archeologici di provenienza di alcuni vasi dipinti con scene di rapimento, sia dalle analogie con i miti di Ganimede e di Core.

In secondo luogo, la serie di raffigurazioni del trasporto di Memnone per opera di Eos alata nella ceramica attica tra la fine del VI e la prima metà del V secolo a.C. è stata messa a confronto con il trasporto del morto Sarpedone compiuto dai gemelli divini Hypnos e Thanatos. Il risalto dato dai pittori al corpo eroico ferito trasportato fuori dal campo di battaglia ha evidenziato l'importanza della morte come tappa indispensabile al raggiungimento della gloria nell'epica omerica e nelle raffigurazioni vascolari del $\mathrm{V}$ secolo a.C. Il tema della morte eroica, enfatizzato dall'intervento divino, si contrappone pertanto a quello della scomparsa anonima, paventata più volte nell'Odissea in riferimento a Odisseo naufrago.

A stabilire un'efficace connessione tra questi due temi - il rapimento di un giovane e il trasporto del corpo di Memnone - di cui Eos appare protagonista nella tradizione epica è il passaggio rappresentato dal movimento della dea alata, interpretabile come transizione da una condizione a un'altra, rispettivamente dall'età infantile a quella adulta nel caso dei rapimenti di giovani, dalla condizione di comune mortale a quella di eroe immortale grazie alla celebrazione da parte della comunità nelle scene di trasporto dell'eroe ucciso in battaglia. 


\section{BIBLIOGRAFIA}

ACETI Chiara, Sarpedone fra mito e poesia, in C. Aceti, D. Leuzzi \& L. Pagani (edd.), Eroi nell'Iliade. Personaggi e strutture narrative, Roma, Edizioni di storia e letteratura, 2008.

ARRIGHETTI Graziano, Esiodo. Opere, testi introdotti, tradotti e commentati da G. Arrighetti; testo greco a fronte, Torino, Einaudi-Gallimard, 1998.

BERGERARD Lorenzo et al., Quinto di Smirne. Il seguito dell'Iliade, coordinamento e revisione di Emanuele Lelli; apparati a cura di V. Zanusso e G. Gagliarde; premessa di G. Cerri, Milano, Bompiani, Il pensiero occidentale, 2013.

BOISACQ Émile, Dictionnaire étymologique de la langue grecque : étudiée dans ses rapports avec les autres langues indo-européennes, Heidelberg, C. Winter, 1938.

BUITRON-OLIVER Diana, Douris: A Master-Painter of Athenian Red-Figure Vases, Mainz,

P. von Zabern, 1995

BURGESS Jonathan S., Early images of Achilles and Memnon?, «Quaderni Urbinati di Cultura Classica», 76 (1), 2004, pp. 33-51.

CALABRESE DE FEO Maria Raffaella, A proposito dell'etimologia delle Arpie, Th. 265-269, «Studi Classici e Orientali», 46 (1), 1996, pp. 331-346.

CAMPAGNOLO Marco, Commento al secondo logos dei Posthomerica di Quinto Smirneo, Università Ca' Foscari di Venezia, OpenAccess, pubblicato il 26 aprile 2012. Disponibile all'indirizzo: <http:// dspace.unive.it/handle/10579/1218> (consultato il 22 dicembre 2017).

CASSOLA Filippo, Inni omerici, a cura di F. Càssola, Milano, Fondazione Lorenzo Valla, 1975.

CENTANNI Monica, Eschilo. Le tragedie, traduzione, introduzioni e commento a cura di M. Centanni, Milano, Mondadori, 2003.

CHANTRAINE Pierre, Dictionnaire étymologique de la langue grecque. Histoire des mots, Paris, Klincksieck, 1968-1980.

COPPOLA Daniela, Anemoi: morfologia dei venti nell'immaginario della Grecia arcaica, Napoli, Liguori, 2010.

COSSU Tatiana, L'arca del tiranno. Umano, disumano e sovrumano nella Grecia arcaica, Cagliari, CUEC, 2009.

CRANE Gregory, Calypso: Backgrounds and Conventions of the Odyssey, Frankfurt am Main, Athenäum, 1988.

DASEN Véronique, Jumeaux, jumelles dans l'antiquité grecque et romaine, Kilchberg, Akanthus, 2005. DEBIASI Andrea, L'epica perduta: Eumelo, il Ciclo, l'occidente, Roma, L'Erma di Bretschneider, 2004 DIHLE Albrecht, Die Griechen und die Fremden, München, C. H. Beck, 1994 (trad. I Greci e il mondo antico, Firenze, Giunti, 1997, da cui si cita).

FERRARI Franco (ed.), Odissea di Omero, Torino, UTET, 2001.

HEUBECK Alfred \& WEST Stephanie, Omero. Odissea, vol. I, Milano, Fondazione Lorenzo Valla, 1981.

JANKO Richard, The Iliad: A Commentary, vol. IV, books 13-16, Cambridge, Cambridge University Press, 1994. 
KAEMPF-DIMITRIADOU Sophia, Die Liebe der Götter in der attischen Kunst des 5. Jahrhunderts v. Chr., Bern, Francke, 1979.

KAEMPF-DIMITRIADOU Sophia, s.v. Boreas, Lexicon Iconographicum Mythologiae Classicae, III (1), Zürich-München, Artemis Verlag, 1986, pp. 133-142.

KANOWSKI Maxwell George, Inscription on the "Memnon Pietà" by Douris, «American Journal of Archaeology», 82, 1978, pp. 549-551.

KNITTLMAYER Brigitte, Die attische Aristokratie und ihre Helden. Darstellungen des Trojanischen Sagenkreises im 6. und frühen 5. Jahrhundert v. Chr., Heidelberg, Verlag Archäologie und Geschichte, 1997.

KOSSATZ-DEISSMANN Anneliese, s.v. Achilleus, Lexicon Iconographicum Mythologiae Classicae, I (1), Zürich-München, Artemis Verlag, 1981, pp. 37-200.

KOSSATZ-DEISSMANN Anneliese, s.v. Memnon, Lexicon Iconographicum Mythologiae Classicae, VI (1), Zürich-München, Artemis Verlag, 1992, pp. 453-455.

LEFKOWTIZ Mary R., “Predatory” Goddesses, Hesperia, 71, 2002, pp. 325-344.

LEWIS Sian, The Athenian Woman: An Iconographic Handbook, London-New York, Routledge, 2002.

LISSARRAGUE François, Corps et armes : figures grecques du guerrier, in J. Wilgaux \& V. Dasen (edd.), Langages et métaphores du corps dans le monde antique, Rennes, Presses universitaires de Rennes, 2015.

LORAUX Nicole, Mourir devant Troie, tomber pour Athènes : de la gloire du héros à l'idée de la cité, in G. Gnoli \& J.-P. Vernant (edd.), La mort, les morts dans les sociétés anciennes, Cambridge-Paris, 1982, pp. 27-43.

MADDOLI Gianfranco, Pausania. Guida della Grecia. Libro V. L'Elide e Olimpia, testo e traduzione a cura di G. Maddoli; commento a cura di G. Maddoli e V. Saladino, Milano, Fondazione Lorenzo Valla, 1995.

NAGY Gregory, Phaethon, Sappho's Phaon, and the White Rock of Leukas, «Harvard Studies in Classical Philology», 77, 1973, pp. 137-177.

NAGY Gregory, The Best of the Achaeans: Concepts of the Hero in Archaic Greek Poetry, BaltimoreLondon, The Johns Hopkins University Press, 1979.

NAGY Gregory, On the Death of Sarpedon, in C. A. Rubino \& C. W. Shelmerdine (edd.), Approaches to Homer, Austin, University of Texas Press, 1983, pp. 189-217.

NEILS Jenifer, The 'Unheroic' Copse: Re-reading the Sarpedon Krater, in J. H. Oakley \& O. Palagia (eds.), Athenian Potters and Painters, vol. II, Oxford, Oxbow Books; Oakville, CT, David Brown Book Company [distributor], 2009, pp. 212-219.

NEUSER Kora, Anemoi. Studien zur Darstellung der Winde und Windgottheiten in der Antike, Roma, G. Bretschneider, 1982.

PADUANO Guido \& MIRTO Maria Serena (edd.), Omero. Iliade, traduzione di G. Paduano; saggi introduttivi di G. Paduano e M. S. Mirto; commento di M. S. Mirto, Torino, Giulio Einaudi Editore, 2012.

PEIFER Egon, Eidola und andere mit dem Sterben verbundene Flügelwesen in der attischen Vasenmalerei in spätarchaischer und klassischer Zeit, Frankfurt am Main, Bern, New York, Paris, Lang, 1997.

PELLIZER Ezio, Tecnica compositiva e struttura genealogica dell'Inno omerico ad Afrodite, «Quaderni Urbinati di Cultura Classica», 27, 1978, pp. 115-144. 
RECKE Matthias, Gewalt und Leid. Das Bild des Krieges bei den Athenern im 6. und 5. Jh. v. Chr., Instanbul, Ege Yayınları, 2002.

RICHARDSON Nicholas, The Iliad: A Commentary, vol. VI, books 21-24, Cambridge, Cambridge University Press, 1993.

RIZZO Salvatore, Viaggio in Grecia. Guida antiquaria e artistica, Libri quinto e sesto: Olimpia ed Elide, introduzione, traduzione e note di S. Rizzo, Milano, Biblioteca Universale Rizzoli, 2001.

ROHDE Erwin, Psyche. Seelencult und Unsterblichkeitsglaube der Griechen, Freiburg im BreisgauLeipzig, J. C. B. Mohr, 1890-1894, tr. it. di E. Codignola e A. Oberdorfer, Prefazione di S. Givone, Roma-Bari, Laterza, 2006.

SCARPI Paolo, Apollodoro. I miti greci, Milano, Fondazione Lorenzo Valla, 1996.

SCHEFOLD Karl, Götter- und Heldensagen der Griechischen in der spätarchaischen Kunst, München, Hirmer, 1978.

SCHNAPP-GOURBEILLON Annie, Les funérailles de Patrocle, in G. Gnoli \& J.-P. Vernant (edd.), La mort, les morts dans les sociétés anciennes, Cambridge, Cambridge University Press / Paris, Maison des sciences de l'homme, 1982, pp. 77-88.

SFORZA Ilaria, L'eroe affidato alle acque: valenze dell'arca dall'epica al mito, «Gaia : revue interdisciplinaire sur la Grèce archaïque», 16, 2013, pp. 211-228.

SFORZA Ilaria, Il peso delle Chere. La Kerostasia in Il. VIII 70 e XXII 210, in I. Baglioni (ed.), Sulle Rive dell'Acheronte. Costruzione e Percezione della Sfera del Post Mortem nel Mediterraneo Antico, vol. 2: L'Antichità Classica e Cristiana, Roma, Edizioni Quasar, 2014, pp. 125-137.

SFORZA Ilaria, Grattandone via la vecchiaia coi suoi saggi espedienti. Nostoi, fr. 7 Bernabè, in E. Calderón Dorda e S. Perea Yébenes (edd.), Estudios sobre el vocabulario religioso griego, Madrid, Salamanca, Signifer Libros, 2016, pp. 187-200.

SFORZA Ilaria, Miasma e condizione eroica nell'Iliade, «Otium», 2 (1), 2017. Disponibile all'indirizzo: $<$ www.otium.unipg.it/otium/article/view/25> (consultato il 20 dicembre 2017).

SHAPIRO Harvey Alan, Personification of Abstract Concepts in Greek Art and Literature to the End of the Fifth Century B.C., Princeton, Princeton University, 1976.

SIMON Erika, s.v. Venti, Lexicon Iconographicum Mythologiae Classicae, VIII (1), Zürich-München, Artemis Verlag, 1997, pp. 186-192.

STANSBURY-O'DONNELL Mark D., Structural Differentiation of Pursuit Scenes, in D. Yatromanolakis (ed.), An Archaeology of Representations: Ancient Greek Vase-Painting and Contemporary Methodologies, Athens, Institut du Livre, A. Kardamitsa, 2009, pp. 342-373.

STEWART Andrew, Rape?, in E. Reeder (ed.), Pandora: Women in Classical Greece, Baltimore, Md., Trustees of the Walters Art Gallery in association with Princeton University Press, Princeton, N.J., 1995, pp. 74-90.

STRAUSS-CLAY Jenny, Sarpédon : Aristos Heroon, «Gaia : revue interdisciplinaire sur la Grèce archaïque», 12, 2009, pp. 15-27.

TSINGARIDA Athena, The Death of Sarpedon: Workshops and Pictorial Experiments, in J. Oakley \& S. Schmidt (edd.), Hermeneutik der Bilder: Beiträge zu Ikonographie und Interpretation griechischer Vasenmalerei («Beihefte zum Corpus vasorum antiquorum», IV), München, C. H. Beck, 2009, pp. 135-142. 
VERMEULE Emily, Aspects of death in early Greek art and poetry, Berkeley, Los Angeles, London, University of California press, 1979.

VERNANT Jean-Pierre, La belle mort et le cadavre outragé, in Id., L'individu, la mort, l'amour. Soi-même et l'autre en Grèce ancienne, Paris, Gallimard, 1989, pp. 41-79.

VON BOTHMER Dietrich, The Death of Sarpedon, in S. L. Hyatt (ed.), The Greek Vase (Papers Based on Lectures Presented to a Symposium Held at Hudson Valley Community College at Troy, New York in April 1979), Latham, Hudson-Mohawk Association, 1981, pp. 63-80.

VON BOTHMER Dietrich, s.v. Sarpedon, Lexicon Iconographicum Mythologiae Graecae, VII (1), ZürichMünchen, Artemis Verlag, 1994.

VIAN Francis, Recherches sur les Posthomerica de Quintus de Smyrne, Paris, Klincksieck, 1959.

WEISS Carina, s.v. Eos, Lexicon Iconographicum Mythologiae Classicae, III (1), Zürich-München, Artemis Verlag, 1986, pp. 747-789.

WEST Martin L., Hesiod. Theogony, edited with Prolegomena and Commentary by M. L. West, Oxford, Clarendon Press 1966.

\section{NOTE}

1. Per un approfondimento delle diverse tipologie di raffigurazioni riferibili a Eos nell'arte greca, si rinvia a Weiss (1986, 747-789): I. Eos als Lichtgottheit; II. Eos im Zusammenwirken mit anderen Göttern; III. Eos und ihre Geliebten; IV. Eos und Memnon.

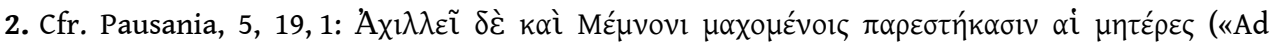
Achille e Memnone intenti a combattere stanno accanto le rispettive madri»). Trad. Maddoli (1995). Sulle più antiche testimonianze figurative del combattimento tra Achille e Memnone nell'arte greca, si veda Burgess (2004, 33-51) e, più in generale, Kossatz-Deissmann (1981, 172-181) e Kossatz-Deissmann (1992, 453-455), con riferimenti bibliografici.

3. Coppa attica a figure rosse, Paris, Louvre G115 (MNB 1698) con iscrizioni su cui cfr. Kanowski (1978, 549-551), firmata dal pittore Duride e dal vasaio Calliade, databile verso il 485/480 a.C.; cfr. LIMC, III (1), s.v. Eos, n. 324, p. 784, con riferimenti bibliografici e infra.

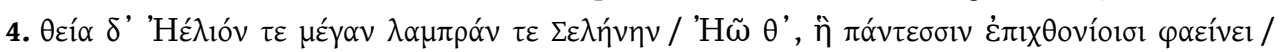

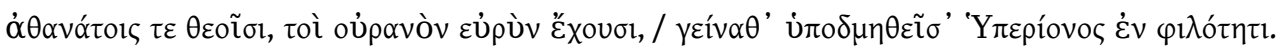
Le traduzioni di Esiodo riportate in questo saggio sono di Arrighetti (1998).

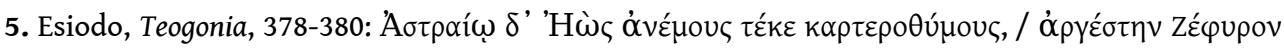

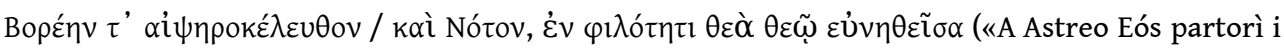
venti dal forte cuore, / lo splendente Zefiro e Borea dalla rapida corsa / e Noto, lei dea con un dio congiunta in amore»).

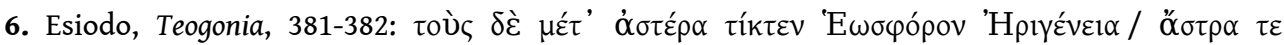

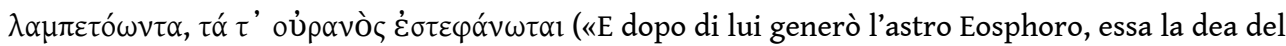
mattino, / e stelle splendenti di cui il cielo è coronato»).

7. Coppola $(2010,95-97)$, spiega la collocazione dei venti divini nati da Eos in una genealogia che si qualifica attraverso la luminosità, come un riferimento alla chiara provenienza del loro soffiare - sebbene non vi sia piena corrispondenza in Esiodo tra questi venti e i quattro punti cardinali,

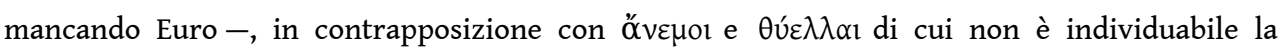
provenienza e per questo associati al buio del Tartaro, sede del mostruoso Tifeo in Esiodo (Teogonia, 869-870: «Da Typheo viene l'umida forza dei venti spiranti, / all'infuori di Noto, Borea e Zefiro splendente»). In merito a questa distinzione originaria tra venti "buoni" provenienti da 
Eos e Astreo e venti "cattivi" provenienti dal Tartaro sede di Tifeo, cfr. inoltre Neuser (1982, 9-10).

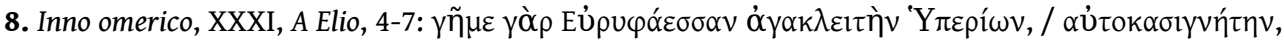

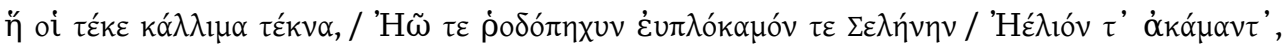

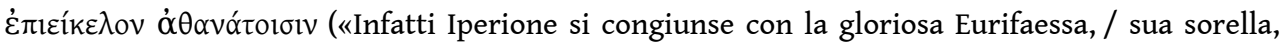
che a lui generò splendidi figli: / l'Aurora dalle braccia di rosa, Selene dalle belle trecce, / ed Elio infaticabile, simile agl'immortali»). Le traduzioni degli Inni omerici riportate in questo saggio sono di Càssola (1975).

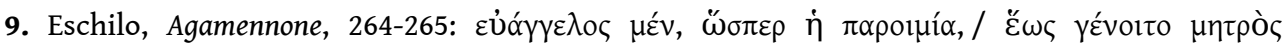

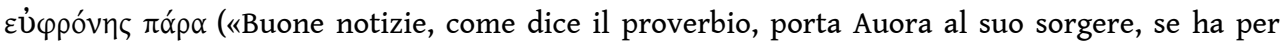
madre una buona notte»). Trad. Centanni (2003).

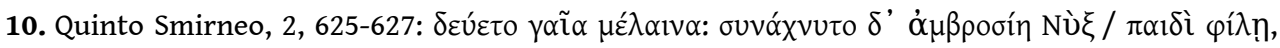

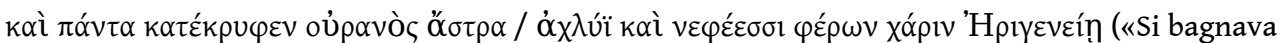
la terra nera; soffriva anche la divina notte / per sua figlia e tutte le stelle coprì Urano / di caligine e nubi, facendo cosa gradita a Erigenia»). Le traduzioni di Quinto Smirneo riportate in questo saggio sono di Bergerard et al. (2013). Per le ipotesi di datazione del poema di Quinto Smirneo, si veda Lelli, Ibidem, Introduzione, XVII-XX.

11. Proclo, Crestomazia, 172 Severyns (= Aethiopis Argumentum, 68-69, 14-15 Bernabè). Per le ipotesi di datazione dell'Etiopide, si veda Debiasi (2004, nota 53, p. 131).

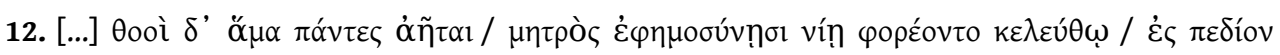

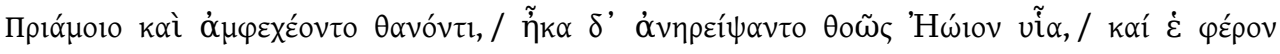

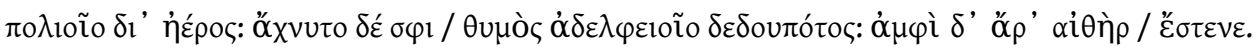

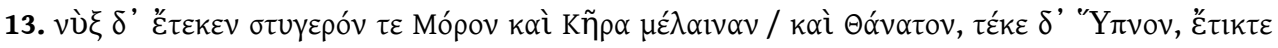

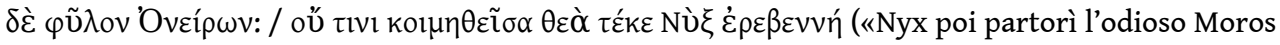
e Ker nera / e Thanatos, generò Hypnos, generò la stirpe degli Oneirata; / non giacendo con alcuno degli dèi li generò Nyx oscura»).

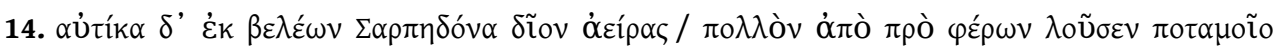

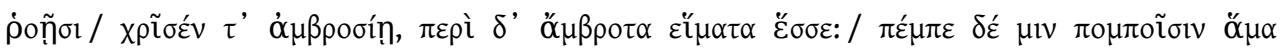

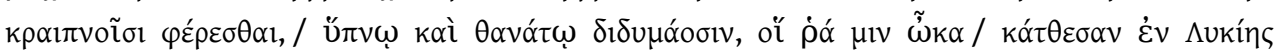

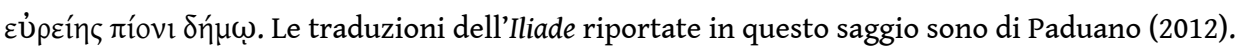

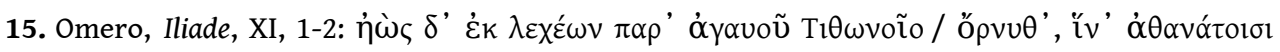

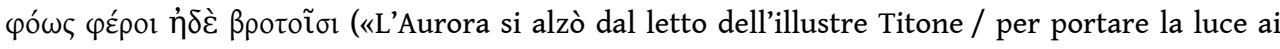

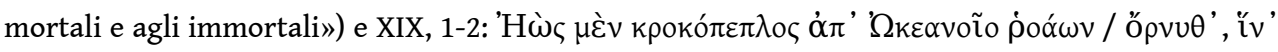

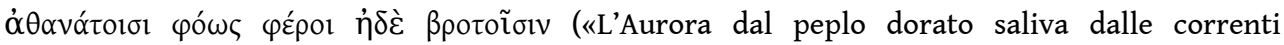
d'Oceano / per portare la luce ai mortali e agli immortali»).

16. Cfr. Vermeule $(1979,163)$ : «In classical Athens the funeral was conducted at night, partly from a reluctance to pollute the day and the living, partly to confirm the release of the soul at dawn. It was natural to express the reason for the practice in mythological terms, that Eos the Dawn goddess carried off the dead "on the wings of the morning", and to motivate the event by simple sexual attraction or love». Per un'approfondita analisi dei rituali funebri nell'Iliade, con particolare riferimento a quelli per Patroclo, si rinvia a Schnapp-Gourbeillon (1982, 77-88); per il significato che tali cerimonie assumono nell'Atene classica, si veda Loraux (1982, 27-43).

17. Omero, Iliade, XXIII, 226-261 e XXIV, 788-803. Per un confronto tra le due scene, si veda Richardson $(1993,196)$ : «But it is striking how much variety there is in the language and ritual details of the two passages [...]» e p. 360: «Verses 791-801 should be compared with 23.250-7 [...]. But the language of the rest is significantly varied, although the basic series of actions is similar». Cfr., inoltre, Omero, Iliade, VII, 433-436, dove, poco prima dell'apparire della luce del giorno, gli Achei, una volta bruciati i corpi dei caduti sui roghi, versano un unico tumulo e innalzano un muro a protezione degli accampamenti e delle navi. 
18. Teogonia, 381.

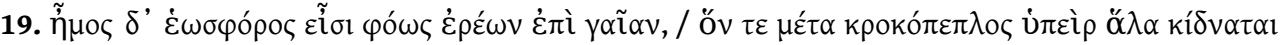

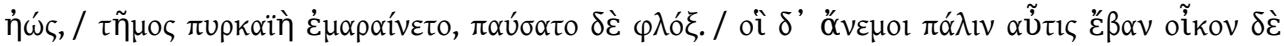

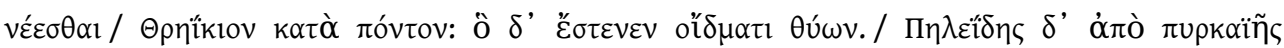

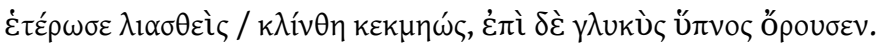

20. Per un confronto tra i due passi e sulla funzione simbolica del contenitore in cui le ossa, rispettivamente di Patroclo e di Ettore, vengono collocate dai compagni, mi si consenta di rinviare a Sforza $(2013,211-228)$.

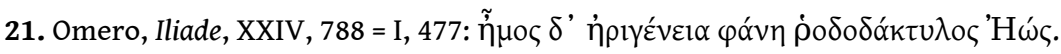

22. Cfr. supra, note 9-10.

23. Per le rare raffigurazioni dei Venti come figure perlopiù alate nell'arte greca, si rinvia a Simon (1997, 186-192); per le rappresentazioni di Hypnos e Thanatos come figure gemellari, spesso caratterizzate contrastivamente come sull'arca di Cipselo (Pausania, 5, 18, 1) e per la loro funzione di portatori del morto sui vasi attici tra il 520 e il 420 a.C., si rinvia a Dasen (2005, 134-136).

24. Omero, Iliade, XXIII, 192-198. Per le diverse interpretazioni date dalla critica all'invocazione dei venti sulla pira di Patroclo, si rinvia a Coppola (2010, 3-8 e passim) con rimandi bibliografici.

25. Omero, Iliade, XXIII, 198-212. Per il carattere inconsueto dell'intervento di Iris, che ha qui il compito insolito di portare a entità divine il messaggio di un mortale, Achille, si vedano le considerazioni espresse da Richardson (1993, 191-192).

26. Cfr. Stansbury-O'Donnell $(2009,358)$ in riferimento alle scene del rapimento di Oreithyia da parte di Borea: «The wings of the god could also be an indicator of his superior power and threat of force, but they can also serve as an index for his divine status, so that we should probably regard the threat of strong force as neutral here».

27. Cfr. infra e Coppola $(2010,12-17)$.

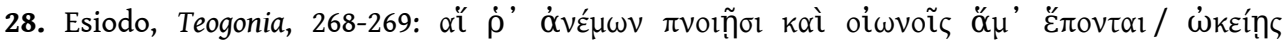

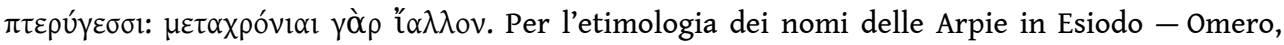

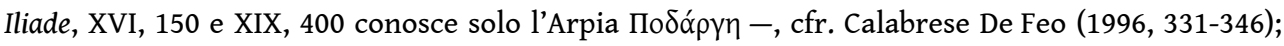
per la loro connessione con Iris, cfr. West $(1966,242):$ «[...] but it is because she (scil. Iris) runs as fast as the winds that the Harpies are joined with her as her sisters».

29. Cfr. Calabrese De Feo (1996, 339-341): «Nel momento in cui Esiodo inserisce le Arpie nella complicata costruzione genealogica della Teogonia [...], è già ben connotata la loro particolare fisionomia di entità vorticose, che si muovono con straordinaria velocità [...]».

30. Cfr. Odissea, I, 241; XVI, 371; XX, 77.

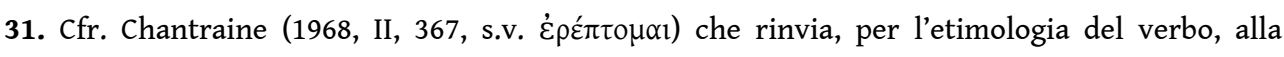
comparazione con il sscr. ap-répti, "afferrare, sollevare" e al lat. rapio con vocalismo zero. Cfr. inoltre Heubeck \& West (1981, 218, ad Od., I, 241): "Appare chiaro che il poeta intese "Ap

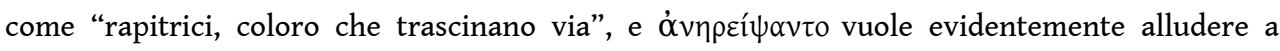
questa etimologia».

32. Karlsruhe, Badisches Landesmuseum inv. B 1904; cfr. Stansbury-O’Donnell (2009, figg. 3-4, pp. 353-356 e 363-364); la provenienza di alcuni vasi con scene di inseguimento da corredi funerari hanno avvalorato l'ipotesi che tali scene rinviino a riti di passaggio, legati a eventi quali la morte o il matrimonio: cfr. Lewis $(2002,205)$.

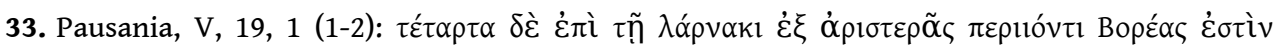

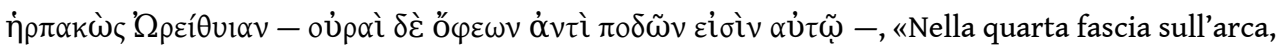
da sinistra, c'è Borea che ha rapito Orizia (al posto dei piedi ha code di serpenti)». Trad. di Cossu $(2009,189)$.

34. Cfr. Cossu (2009, 189-191, con riferimenti bibliografici). 
35. Sul tema del "rapimento" di un mortale da parte di una dea nelle attestazioni letterarie cfr. Nagy (1973, 156 e ss.) e Nagy (1979, Ch. 10: Poetic Visions of Immortality for the Hero, 174-210). Sulla fortuna iconografica di questo tema, si vedano inoltre Vermeule (1979, 145-178), KaempfDimitriadou (1979), Stewart (1995, 74-90), Lefkowtiz (2002, 325-344) e, da ultimo, StansburyO'Donnell (2009, 342-344), con ulteriore bibliografia.

36. Cfr. per es. Odissea, II, 1 = Iliade, XXIV, $788=$ I, 477, ecc.

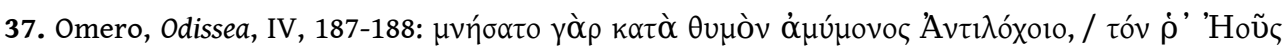

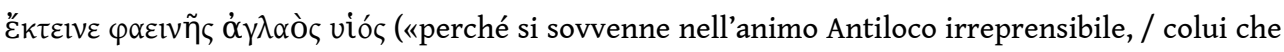
il figlio radioso della fulgida Aurora aveva ucciso»). Le traduzioni dell'odissea riportate in questo saggio sono di Ferrari (2001). L'episodio faceva parte dell'Etiopide (Proclo, Crestomazia, 172 Sev. =

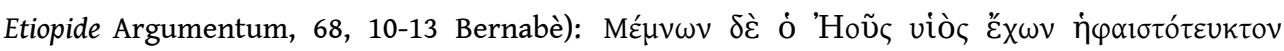

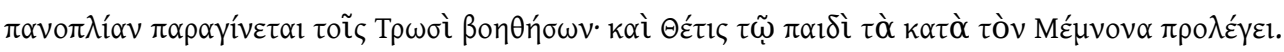

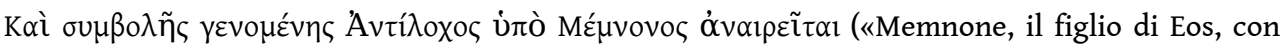
una corazza fabbricatagli da Efesto viene in soccorso dei Troiani e Teti predice al figlio i fatti relativi a Memnone. Poi, durante un combattimento, Antiloco è ucciso da Memnone»). Cfr.,

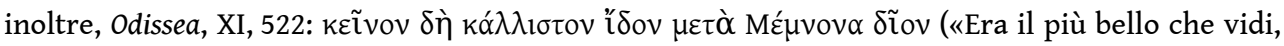
dopo il nobile Memnone»).

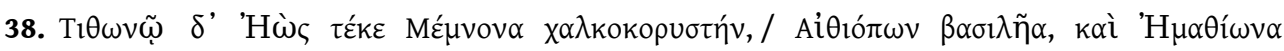

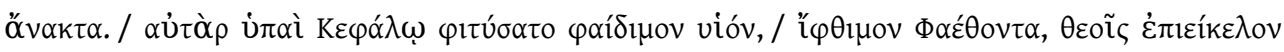

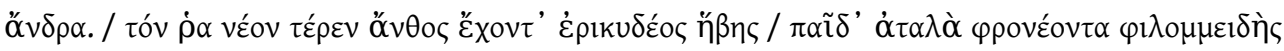

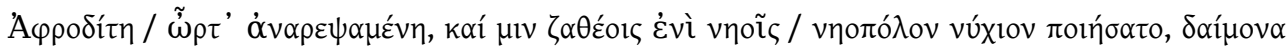

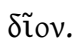

39. Per le ipotesi etimologiche qui suggerite e per altre ipotesi possibili, si rinvia al Dizionario Etimologico della Mitologia Greca Online, s.v. Memnone [in linea]: <https://demgol.units.it/lemma.do? id=827>; ed Emazione: <https://demgol.units.it/lemma.do?id=859> (consultato il 20 dicembre 2017).

40. Sul significato assunto dalla consacrazione di Fetonte a custode e genio divino da parte di Afrodite, si vedano le considerazioni espresse da Nagy $(1979,191)$ : «The designation of Phaethon as daímōn also conveys the immortal aspect of the hero in his afterlife, since it puts him in the same category as the Golden Generation, who are themselves explicitly daímones (Works and Days, 122)».

41. Per il rapimento di un mortale da parte di un'entità divina che lo sottrae così al suo destino naturale, relegandolo in una dimensione altra, posta ai margini dell'ecumene abitata dagli uomini, dove conduce una vita eterna in tutto simile a quella degli dei, si veda Rohde (2006, 65-97).

42. Omero, Odissea, $\mathrm{V}, 1-2$ (= Iliade, XI, 1-2).

43. Inno omerico, $\mathrm{V}$, Ad Afrodite, 218-238. Per il significato assunto qui dall'espressione छ̃̃ $\sigma \alpha i ́ ~ \tau '$

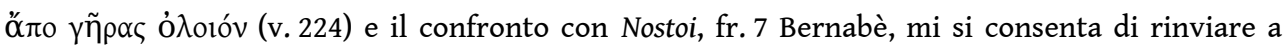
Sforza $(2016,187-200)$.

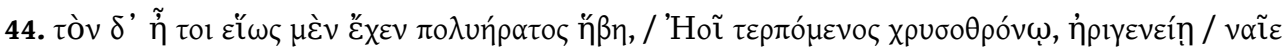

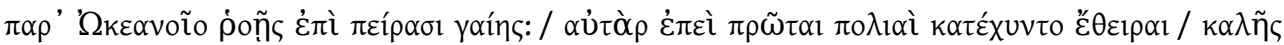

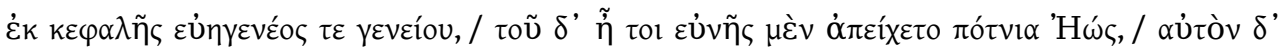

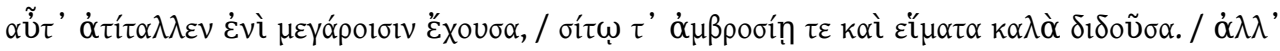

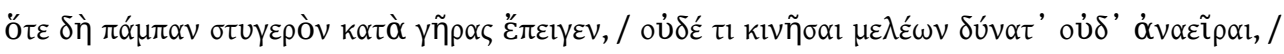

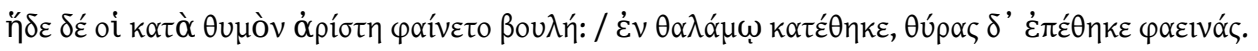

45. Proclo, Crestomazia, 172 Severyns = Etiopide, Argumentum, 68-69, 14-15 Bernabè: ع̌ $\pi \varepsilon ı \tau \alpha$

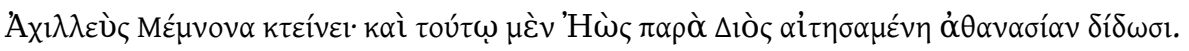

46. Omero, Iliade, XVI, 676-683; cfr. supra. 


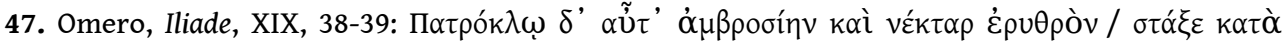

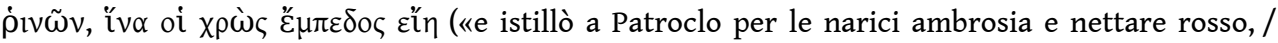
perché il suo corpo restasse intatto»).

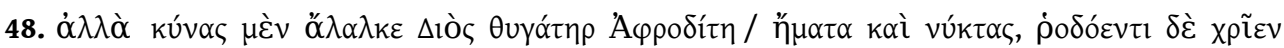

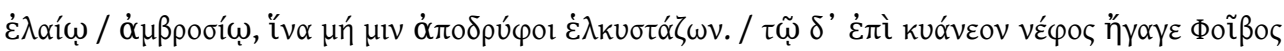

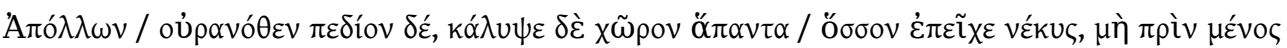

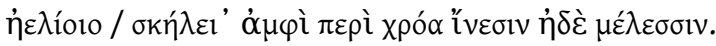

49. Per il valore di queste operazioni simboliche volte a preservare il volto dell'eroe nella belle mort di cui parla Vernant $(1989,41-79)$ e per il valore inverso della contaminazione causata dalla polvere e dal sangue, si rinvia alle considerazioni espresse in Sforza (2017).

50. Eschilo, Tragicorum Graecorum Fragmenta, III, F 279-280a Radt. Sulla psicostasia associata al duello tra Achille e Memnone nelle immagini, cfr. A. Kossatz-Deissmann (1992, nn. 14-25, 451-453). Per un confronto tra la psicostasia di Achille e Memnone nell'Etiopide e le due scene di "kerostasia" dell'Iliade, mi si consenta di rinviare a Sforza (2014, 125-137).

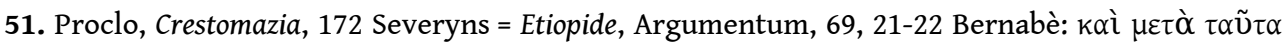

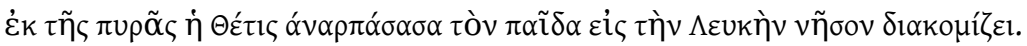

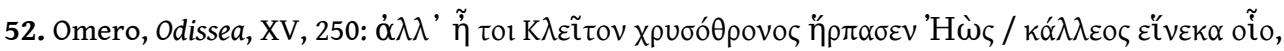

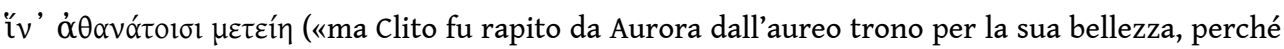
vivesse fra gli eterni»).

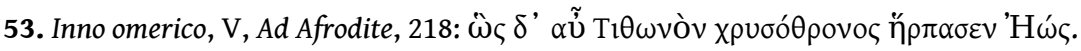

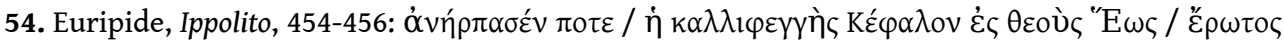

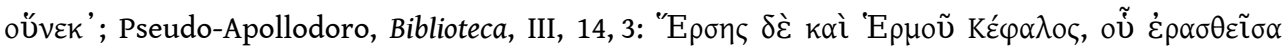

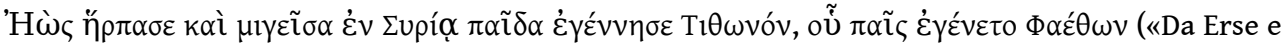
da Ermes nacque Cefalo; di lui si innamorò Eos che lo rapì e gli si unì in Siria, generando il figlio Titono, da cui nacque Fetonte»).

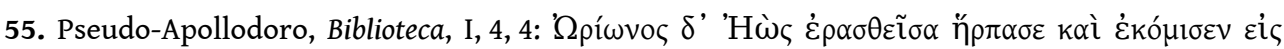

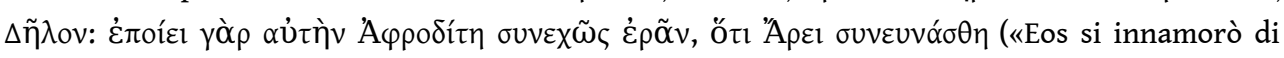
Orione, lo rapì e lo portò a Delo: Afrodite infatti faceva sì che Eos si innamorasse continuamente, perché era andata a letto con Ares»). Trad. Scarpi (1996).

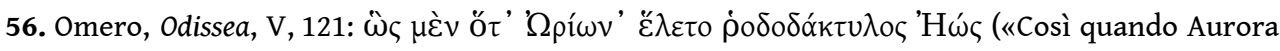

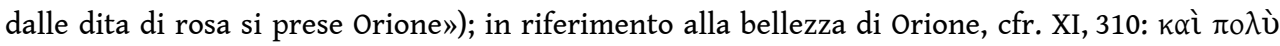
$\kappa \alpha \lambda \lambda i ́ \sigma \tau o u \varsigma ~ \mu \varepsilon \tau \alpha^{\prime} \gamma \varepsilon \kappa \lambda u \tau o ̀ v ~ ' \Omega \rho i ́ \omega v \alpha$ («[...] e i più belli che dopo Orione glorioso nutrì [...]»), e alla

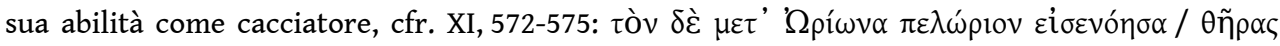

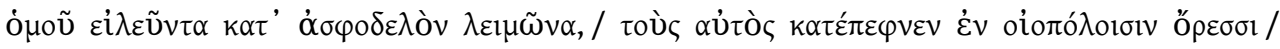

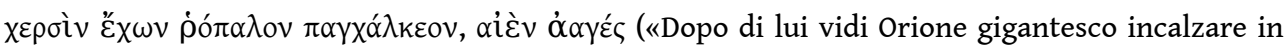
branco le fiere per il prato di asfodelo, quelle che uccideva sui monti solitari impugnando una mazza infrangibile di bronzo massiccio»). Per le diverse cause della morte di Orione, decritto a sua volta come un amante insaziabile e un violento cacciatore nelle fonti antiche, si veda Scarpi (1996, 441-442).

57. Per il valore escatologico dell'intero episodio del soggiorno di Odisseo a Ogigia e un confronto tra il canto V dell'Odissea e l'Inno omerico, II, A Demetra, si rinvia a Crane (1988, 16-21).

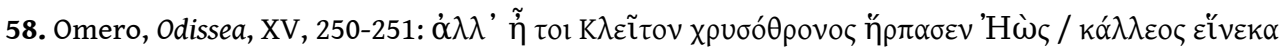

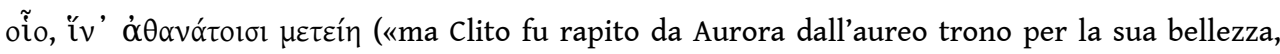
perché vivesse fra gli eterni»).

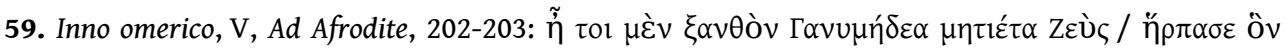

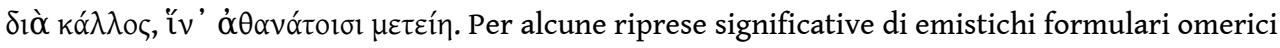
in questo inno, si rinvia a Pellizer (1978, 115-144). 


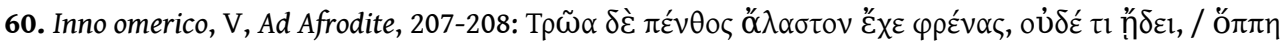

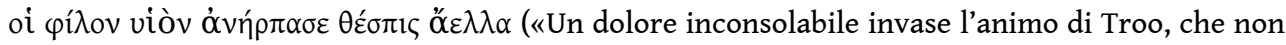
sapeva / dove il turbine divino gli avesse rapito suo figlio»).

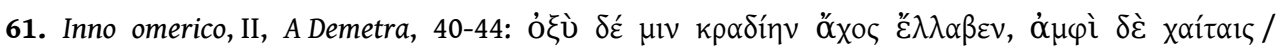

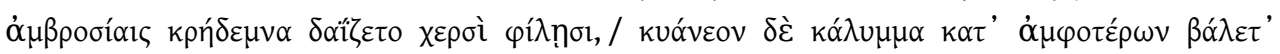

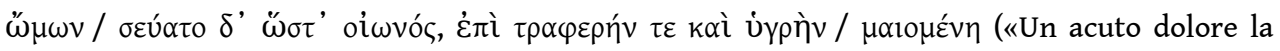
colse nell'animo: sulle chiome / divine lacerava con le sue mani il diadema, si gettava sulle spalle un cupo velo / e si slanciò sopra la terra e il mare, come un uccello, / alla ricerca»).

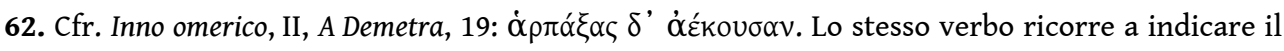

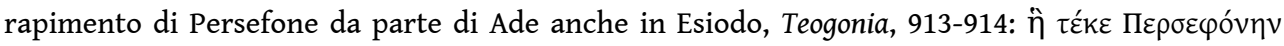

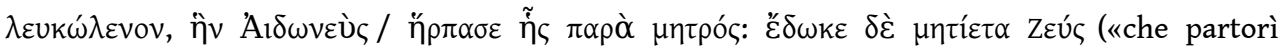
Persefone dalle bianche braccia; Adoneo costei / rapì da sua madre: a lui la concesse Zeus prudente»).

63. Omero, Odissea, XX, 66.

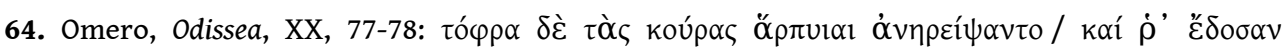

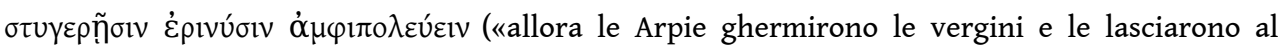
servizio delle odiose Erinni»).

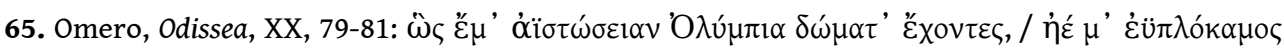

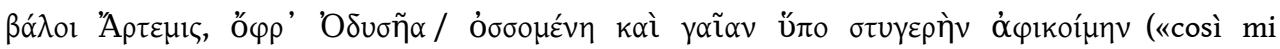
annichilissero coloro che abitano le case d'olimpo o mi colpisse Artemide dai riccioli belli, sì che io scendessi all'aborrita contrada serbando negli occhi il mio Odisseo»).

66. Cfr. Odissea, IV, 515; XX, 63; XXIII, 316 ecc.

67. Si vedano, in proposito, le opportune riflessioni di Nagy $(1983,202)$ : «Homeric poetry tends not to speak in a direct fashion about immortalization because Homeric poetry presents itself as the very process of immortalization».

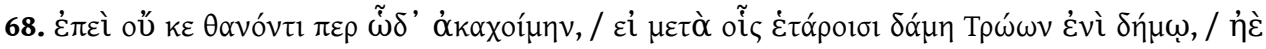

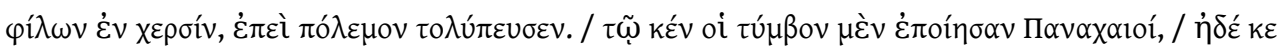

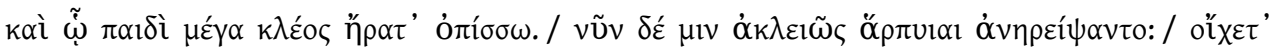

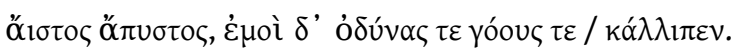

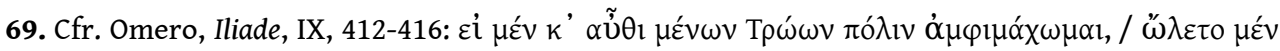

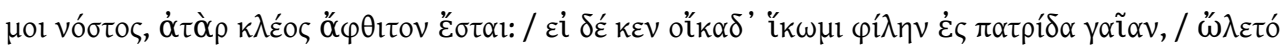

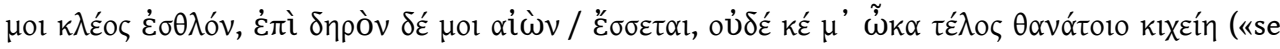
resto qui a combattere attorno alla città dei Troiani, / è perduto per me il ritorno, ma avrò gloria immortale: / se invece torno a casa, alla mia patria, / è perduta per me la nobile gloria, ma la mia vita / durerà a lungo e la morte non mi colpirà così presto») e il commento di Nagy (1973, 175-176).

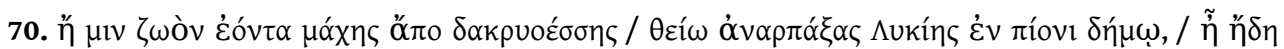

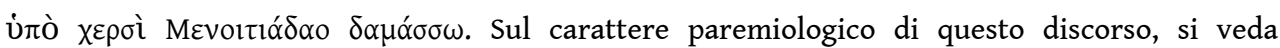
Aceti $(2008,99-113)$ con riferimenti bibliografici.

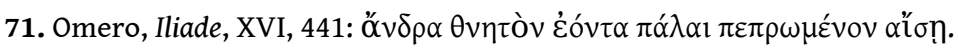

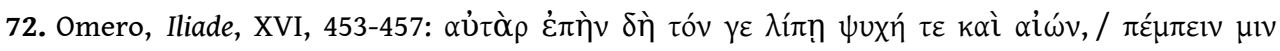

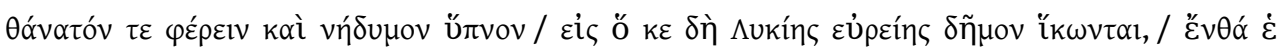

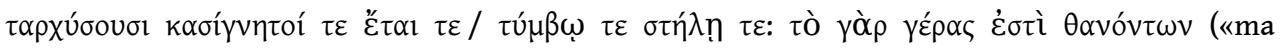
appena / lo avrà abbandonato il respiro e la vita, / manda a prenderlo la Morte e il Sonno soave, / che vadano nel paese dell'ampia Licia, / dove parenti ed amici lo seppelliranno e gli daranno / una tomba e una stele: è questo l'onore dei morti»). Per la problematica interpretazione in questo

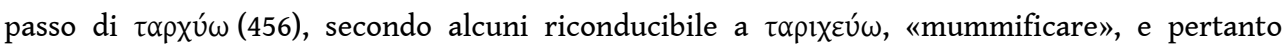
allusivo a una pratica dell'imbalsamazione del cadavere non altrimenti attestata nei poemi 


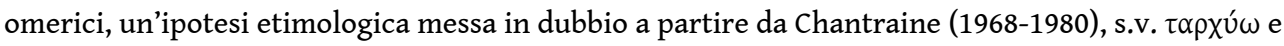
ritenuta inattendibile da Janko (1994, 377), cfr. Aceti (2008, nota 252, pp. 110-111).

73. Per una retrospettiva critica e per l'influsso delle tesi neoanalitiche anche su commentatori come Janko (1994, 372), si veda Aceti $(2008,232)$.

74. Il legame tra questi due concetti appare confermato dall'etimologia popolare che riconduce il nome di Sarpedone a quello delle Arpie: cfr. Boisacq $(1938,81)$; per altre ipotesi etimologiche, che tuttavia, non sembrano confermate dalle vicende di cui Sarpedone è protagonista nell'epica omerica, si veda il Dizionario Etimologico della Mitologia Greca Online, s.v. Sarpedone: <https:// demgol.units.it/lemma.do?id=33> (consultato il 20 dicembre 2017). Sul rapporto tra Sarpedone e le Arpie nel culto, si veda inoltre Vermeule $(1979,169)$; più in generale, sulla figura di Sarpedone e l'ideologia del klèos àphthiton di cui questo personaggio si fa portatore nel poema, cfr. StraussClay (2009, 22): «La mortalité individuelle ne peut être dépassée - paradoxalement - que par le courage personnel, par la confrontation avec la mort, et finalement par la mort hérö̈que. La récompense est le kleos impérissable, une forme de mémoire qui à la fois transcende la mortalité et constitue son seul antidote».

75. In proposito, si vedano soprattutto Von Bothmer (1981, 63-80), Shapiro (1976, 129-136) e, più di recente, Tsingarida $(2009,135-142)$.

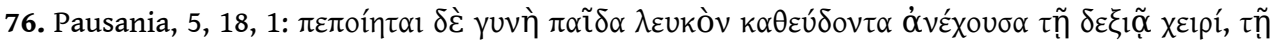

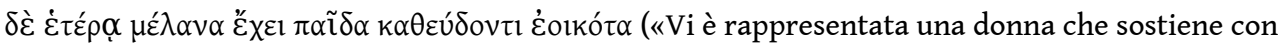
la destra un bambino bianco addormentato e con la sinistra un bambino nero in atteggiamento di dormiente». Trad. Rizzo (2001).

77. Per un'analisi delle scene di trasporto di Sarpedone da parte di Hypnos e Thanatos cfr. Von Bothmer (1994, nn. 3-15, pp.697-698), Peifer (1997, 212-234) e Recke (2002, 60-64; cat. p. 288, tavv. 42-43), con ulteriori riferimenti bibliografici.

78. Per una discussione delle raffigurazioni in cui Eos alata trasporta Memnone, si rinvia a Weiss (1986, nn. 317-326, pp. 783-784), Peifer (1997, 199-209) e Recke (2002, 64-68, cat. 289-290; tavv. 44-47), con ulteriore bibliografia. Per la contestualizzazione di tali scene nel più ampio tema epico del trasporto del morto, cfr. Knittlmayer (1997, 57-60).

79. Paris, Louvre CA4201; cfr. LIMC, III (1), s.v. Eos, n. 328, p. 784; cfr. Peifer (1997, K127, p. 205) e Recke (2002, 289, n. 2, tav. 44b).

80. Pfeifer (1997, 205): «Sie hält den Körper, wie man ein Kind zu halten pflegt, mit Griff unter den Schulterblättern und unter dem Gesäß».

81. Su una lekythos a figure rosse databile verso il 470 a.C., conservata a Madrid, Museo Arqueologico Nacional 11158 (fig. 1), per esempio una figura femminile alata sovradimensionata trasporta verso destra un giovane nudo con in mano una lira; l'immagine viene solitamente interpretata come Eos che rapisce Cefalo o Titono: cfr. LIMC, III (1), s.v. Eos, n. 268, p. 773; un analogo schema è rappresentato su uno skyphos a figure rosse databile verso il 450/440 a.C. conservato a Cambridge, Corpus Christi College T520 b2; cfr. LIMC, III (1), s.v. Eos, n. 271, p. 773 e su un altro skyphos a figure rosse contemporaneo conservato a Firenze, Museo Archeologico Etrusco 4228; cfr. LIMC, III (1), s.v. Eos, n. 272, pp. 773-774: in entrambe le immagini il giovane è nudo e porta in mano una lira.

82. Per l'interpretazione funeraria delle scene di rapimento, si vedano Vermeule (1979, $163 \mathrm{ss}$.), Lewis (2002, 199-205), Lefkowtiz (2002, 325-344) e, da ultimo, Stansbury-O'Donnell (2009, 370-371): «[...] along with an erotic/gender inversion reading of the Eos/Kephalos image, we should consider a concurrent funerary meaning for the image as a result of the divine and threat dimensions of the image's structure».

83. Bochum, Ruhr Universitat, Kunstsammlungen S494; cfr. LIMC, III (1), s.v. Eos, n. 318, p. 783; Recke (2002, Eos und toter Memnon, n. 5, p. 65, tav. 45d). Uno schema simile si riscontra su uno skyphos a figure nere dello stesso periodo, conservato Paris, Louvre CA1812; cfr. Recke (2002, Eos und toter Memnon, nn. 9-10), dove il morto ha la barba e i capelli lunghi e, accanto alle due figure principali, è raffigurato un vecchio stante con il bastone, che assiste alla scena. 
84. Berlin F2318, da Vulci; cfr. LIMC, III (1), s.v. Eos, n. 322, p. 784; Recke (2002, Eos und toter Memnon, n. 19, p. 66, tav. 46b). Per le diverse identificazioni della figura femminile proposte - la moglie di Memnone, Teti, la madre di Achille ecc. -, si rinvia a Peifer (1997, K123, p. 202 e nota 446).

85. Paris, Musée du Louvre G232; cfr. LIMC, III (1), s.v. Eos, n. 332, p. 785. Anche sul noto cratere a figure rosse del pittore Eucharides, Paris, Louvre G163, databile verso il 500-480, sia le figure dei due portatori alati, sia il morto, hanno un aspetto giovanile; cfr. Recke (2002, Hypnos und Thanatos, n. 8, p. 63, tav. 43c) e il commento di Vermeule (1979, p. 150, fig. 3): «When Sleep and Death are young, the body often seems too heavy for them to lift [...]».

86. Paris, Musée du Louvre G115 (MNB 1698): cfr. LIMC, III (1), s.v. Eos, n. 324, p. 784; Recke (2002, Eos und toter Memnon, n. 22, p. 66, tav. 46a); Buitron-Oliver (1995, n. 119, tav. 71, commento a p. 31): «Already dead, his body has become almost weightless, having lost the life force that bound it to the ground. The powerful wings of Eos, lifted, skimming the tondo border, suggest her imminent flight to carry her son home to Africa».

87. Cratere a figure rosse, databile verso il 515-510 a.C., firmato dal pittore Euphronios, exNew York, Metropolitan Museum of Art 1972.11.10, prestato alla Repubblica Italiana (L.2006.10); cfr. LIMC, VII (1), s.v. Sarpedon, n. 4, p. 697 con riferimenti bibliografici.

88. Le profonde ferite che segnano il corpo nudo e privo di vita di Sarpedone sul cratere a figure rosse di Euphronios ricordano le offese arrecate al corpo del nemico ucciso dai Greci, allo scopo di offenderne la bellezza e di disonorarlo: cfr. Neils (2009, 212-219) e Recke (2002, Hypnos und Thanatos, n. 6, tav. 42b, commento a p. 62).

89. Anfora a figure nere databile tra il 540 e il 520 a.C., Roma, Vaticano 16589; ABV 140/1; cfr. LIMC, III (1), s.v. Eos, n. 327, p. 784 con riferimenti bibliografici; per l'interpretazione della scena, cfr. Recke (2002, p. 289, n. 1, tav. 44a e p. 65): «Die Deutung der Szene hängt auch an dem Vogel, der über der Frau im Geäst sitzt und in dem gegebenenfalls ein sogenannter Memnonsvogel erkannt werden darf. Gegen die Benennung der beiden Figuren als Eos und ihr toter Sohn Memnon spricht dabei hauptsächlich die Gestalt der Frau, die flügellos dargestellt ist».

90. L'ipotesi è sostenuta, tra gli altri, da Schefold (1978, 244, fig. 328).

91. Per la disposizione delle armi del morto, cfr. Lissarrague (2015, 15-27, fig. 3, p. 10): «Les armes sont disposées comme un trophée, non pas en tas, mais verticalement, contrastant ainsi avec l'horizontalité du cadavre. Les deux parties se séparent, et la vitalité semble perdurer dans l'armure opposée à l'inertie du cadavre».

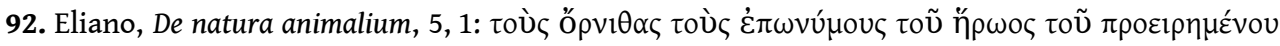

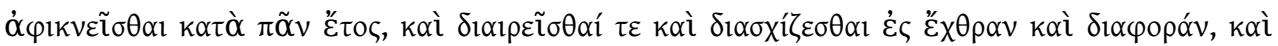

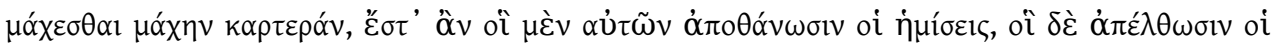

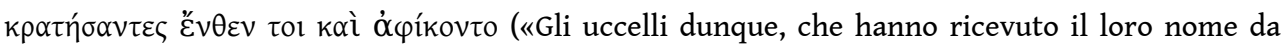
detto eroe, si recano ogni anno sul luogo, il loro battaglione si separa in due e si divide in due fazioni ostili e opposte, le quali si scagliano in un combattimento violento finché i membri di uno dei due schieramenti muoiono e gli uccelli dell'altro gruppo, vittoriosi, se ne vanno e ritornano da dove sono venuti») e Lattanzio Placido, Narrat. Fabul. Ovid. Met., XIII, fab. 3, p. 701 Magnus (= Fragmenta Hesiodea 353 Merkelbach-West), che attribuisce a Esiodo la seguente notizia: Memnon, Tithoni et Aurorae filius, Priamo ferens auxilium ab Achille occiditur. Mater ergo precibus pro assiduo officio inducendae lucis ab Iove impetrat, ut in cineres eius adusto rogo sorores convertantur in volucres Memnonides nomine. Quae memores belli cum quotannis ad sepulcrum eius conveniunt, et inter se dimicantes sanguine suo manibus eius frequentia parentant et ipsa mater eius matutinis temporibus lacrimas desiderio filii sui Memnonis transmutat in rorem. Quod tamen monumentum in Phrygia constituit patruus eius, ut Hesiodus vult.

93. Quinto Smirneo, 2, 556-566: «Ma quante gocce del suo sangue caddero a terra / dalle membra, tra gli uomini esse sono diventate un luogo di ricordo $(\sigma \tilde{\eta} \mu \alpha)$, anche per i posteri; gli dei infatti qua e là / raccoltele in un sol luogo, ne fecero un fiume risonante, / quello che Paflagonio 
chiamano i mortali, / tutti quanti abitano alle pendici dell'alto Ida; / questo fiume scorre sulla terra feconda rosso sangue, / ogni volta che torna il triste giorno di Memnone, quello in cui egli / morì: penoso e insopportabile odore vien su / dall'acqua; diresti che ancora dalla ferita funesta / esali sangue imputridito, molesto».

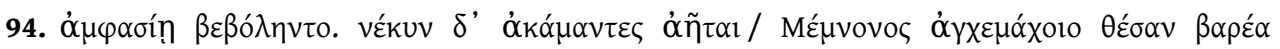

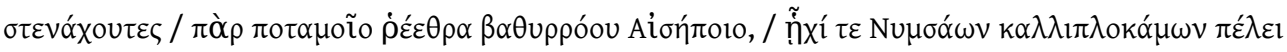

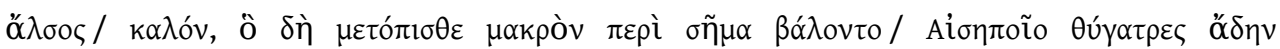

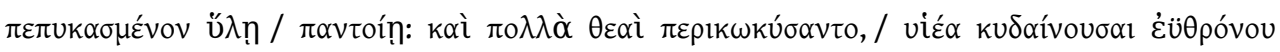
'H prүeveíns.

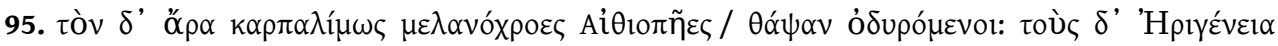

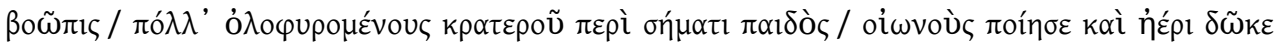

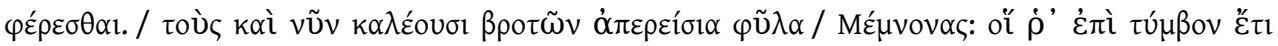

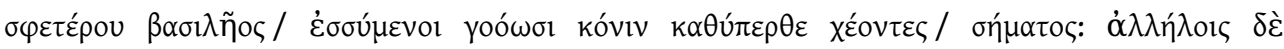

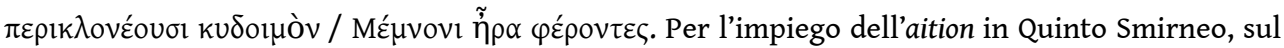
modello dei poeti ellenistici, in primis Callimaco e Apollonio Rodio, cfr. Campagnolo (2012, 37-40). Per la trasformazione degli Etiopi in uccelli vedi anche Ovidio, Metamorfosi, 13, $607 \mathrm{ss.} \mathrm{Per} \mathrm{la}$ versione del racconto in Quinto Smirneo, probabilmente esemplata su un passo dei perduti Ixeutika di Dionisio il Periegeta di cui disponiamo di una parafrasi in prosa, cfr. Vian (1959, 28-30).

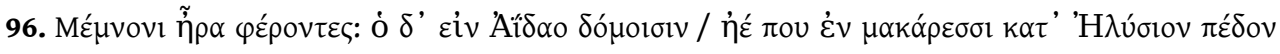

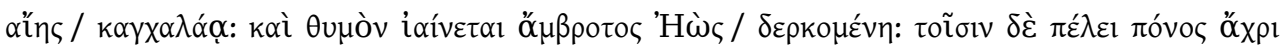

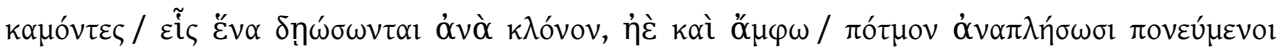

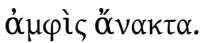

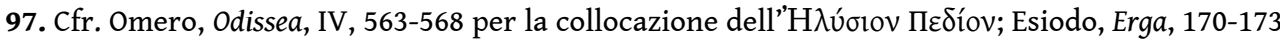

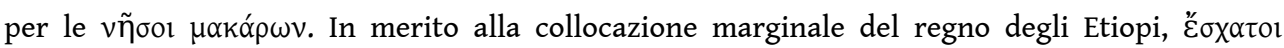
$\dot{\alpha} v \delta \rho \tilde{\omega} v$, cfr. Omero, Odissea, I, 23-24; per un commento sulla "marginalità" del regno di Memnone nelle fonti epiche, cfr. Nagy (1979, 205-208); sull'effettiva conoscenza degli Etiopi già da parte dei micenei, testimoniata dalla presenza del nome ai-ti-jo-ko sulle tavolette scritte in lineare B del palazzo di Pilo (XIII sec. a.C.), cfr. Dihle (1997, 7-10).

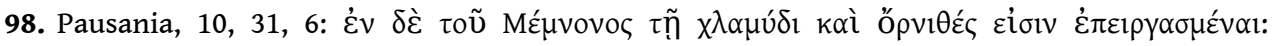

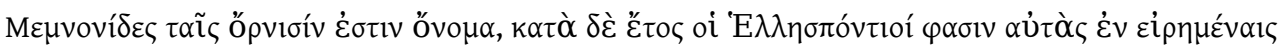

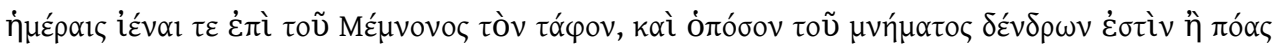

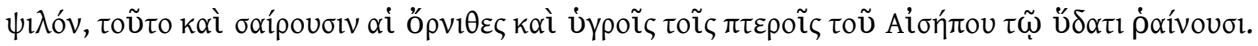

\section{RIASSUNTI}

In questo contributo conduciamo un'indagine sulle funzioni di Eos con particolare riferimento all'epica omerica, proponendo un confronto con alcune raffigurazioni della dea nella ceramica vascolare del V secolo a.C. Nelle fonti letterarie come in quelle iconografiche risalta l'associazione di Eos con il tema del rapimento di alcuni giovinetti variamente identificati con Titono, Orione, Clito, Cefalo. All'esame di alcuni passi omerici questo tema è connotato negativamente per quegli eroi che un dio vorrebbe sottrarre al combattimento preservandoli dalla morte. Nelle immagini si delinea per antitesi il valore positivo della morte eroica, esaltato nelle scene del trasporto di Sarpedone da parte di Hypnos e Thanatos. In conclusione, il sollevamento del corpo di Memnone da parte della dea alata è interpretabile come un 
cambiamento di status, grazie al raggiungimento dell'eroe, dopo la morte, di una gloria immortale.

Dans cette contribution, nous menons une enquête sur les fonctions d'Éos en portant une attention particulière à l'épopée homérique et en proposant une confrontation avec quelques représentations de la déesse dans la céramique vasculaire du ve siècle av. J.-C. L'examen des sources littéraires aussi bien qu'iconographiques montre une association fréquente d'Éos avec la thématique de l'enlèvement de quelques jeunes gens identifiés tantôt à Tithon, tantôt à Orion, Kleitos ou Képhalos. L'examen de quelques passages homériques révèle que ce thème est connoté de façon négative pour les héros qu'un dieu voudrait soustraire au combat en les préservant de la mort. L'iconographie souligne par antithèse la valeur positive de la mort héroïque, exaltée dans les scènes de transport de Sarpédon par Hypnos et Thanatos. Il faudrait donc interpréter le soulèvement du corps de Memnon par la déesse ailée comme un changement de statut, qui permet au héros d'atteindre, après sa mort, une gloire immortelle.

\section{INDICE}

Mots-clés : mythologie grecque, épique homérique, céramique attique, littérature grecque

Parole chiave : mitologia greca, epica omerica, ceramica attica, letteratura greca

\section{AUTORE}

\section{ILARIA SFORZA}

Università degli Studi di Roma «Tor Vergata».

Ilaria Sforza a étudié à l'École normale supérieure de Pise et à l'École des hautes études en sciences sociales à Paris. Elle est membre du comité de rédaction de la revue Rationes Rerum Rivista di Filologia e Storia (université de Rome «La Sapienza» et «Tor Vergata»). Elle est l'auteure de la monographie L'eroe e il suo doppio (2007) et de l'édition italienne de San Ticone (2007) et de Le storie del diluvio (2010) du philologue et historien des religions allemand Hermann Usener. Elle prépare l'édition critique de l'historien hellénistique Hécatée d'Abdère pour la série «I Frammenti degli Storici Greci» (FStGr) de l'université de Rome «Tor Vergata». 